\title{
THE DEPENDENCE ON PARAMETERS OF THE INVERSE FUNCTOR TO THE $K$-FINITE FUNCTOR
}

\author{
NOLAN R. WALLACH
}

Abstract. An interpretation of the Casselman-Wallach Theorem is that the $K$-finite functor is an isomorphism of categories from the category of finitely generated, admissible smooth Fréchet modules of moderate growth to the category of Harish-Chandra modules for a real reductive group, $G$ (here $K$ is a maximal compact subgroup of $G$ ). In this paper we study the dependence of the inverse functor to the $K$-finite functor on parameters. Our main result implies that holomorphic dependence implies holomorphic dependence. The work uses results from the excellent thesis of van der Noort. Also a remarkable family of universal Harish-Chandra modules, developed in this paper, plays a key role.

\section{INTRODUCTION}

The Casselman-Wallach (C-W) Theorem implies that the $K$-finite functor is an isomorphism of categories from the category of finitely generated, admissible smooth Fréchet modules of moderate growth to the category of Harish-Chandra modules for a real reductive group, $G$ (here $K$ is a maximal compact subgroup of $G$ ). This variant will be explained in detail in Section 2 since the usual interpretation is that the $\mathrm{C}-\mathrm{W}$ theorem is an equivalence of categories. A description of the inverse functor, $V \rightarrow \bar{V}$, to the $K$-finite functor is described therein. In this paper we study the dependence of this functor on parameters. Our main result implies that holomorphic dependence implies holomorphic dependence (see Theorem 9.2 and Appendices $\mathrm{D}$ and $\mathrm{F}$ for the pertinent definitions). This work rests on the excellent thesis of Vincent van der Noort $[\mathrm{VdN}]$ which contains several remarkable theorems including his finiteness theorem that is given a slightly simplified proof in Appendix E. In his thesis van der Noort proved a version of the main theorem of this paper for one complex dimensional parameter spaces (see $[\mathrm{VdN}$, Chapter 6$]$ ). In the final section (6.5) he laid out a scheme to prove that two holomorphic families of Fréchet completions of moderate growth yielding the same holomorphic family of HarishChandra modules are equal. This equality is a consequence of our main theorem. In addition to van der Noort's results our technique involves the study of a class of standard modules in the Harish-Chandra category with remarkable properties. Which for lack of a name we call $J$-modules. In particular, they are free modules for the universal enveloping algebra of a maximal unipotent subgroup of $G$. Also, every Harish-Chandra module has a resolution by these modules.

The technical general results not specific to the main results of this paper are the content of the many appendices which take up more space than the body of

\footnotetext{
Received by the editors February 5, 2021, and, in revised form, August 3, 2021, August 24, 2021, and September 10, 2021.

2020 Mathematics Subject Classification. Primary 22E45, 22E30.
} 
the paper. Hopefully this separation will help the reader see the flow of the proof of the main theorem which is quite intricate (we give a brief sketch of it in the next paragraph). For example, the reader could, on first reading, skip the proofs in the appendices. Among the appendices there are results that are of interest beyond this paper. For example, Appendix $\mathrm{A}$ gives a proof that the $C^{\infty}$ vectors relative to $G$ of a finitely generated, admissible Hilbert representation are the same as the $C^{\infty}$ vectors relative to $K$ (see Proposition A.2).

0.1. The organization of the paper and a sketch of the proof of the main theorem. The first section sets up the notation and the class of groups that will be studied in this paper. The second gives a natural description of the so-called Casselman-Wallach globalization of a Harish-Chandra module, $V$, for a real reductive group (see Sections 1 and 2 for pertinent definitions). This construction defines $T: V \rightarrow \bar{V}$ a functor from the category of Harish-Chandra modules to the category of admissible, smooth Fréchet representations of moderate growth which is inverse to the $K$-finite functor (see Theorem 2.2 and the paragraph following its statement). The aim of the paper is to show that applying $T$ to a holomorphic family of Harish-Chandra modules yields a holomorphic family of smooth Fréchet representations of moderate growth. To implement the goal I first construct and study the analytic and algebraic properties of the class of Harish-Chandra modules, alluded to above as $J$-modules. This occupies the next 5 sections of the paper. I show that if one has an analytic family of Harish-Chandra modules (see Appendix D) and if $U$ is an open set with compact closure in the parameter space there is a family of $J$-modules over $U$ mapping surjectively onto the restriction of the family to $U$. The next step is to locally (in the parameter) globalize a continuous family of $J$-modules to a continuous family of Hilbert representations satisfying a technical condition (smoothable) that implies that the corresponding family of $C^{\infty}$-vectors defines a continuous family of smooth Fréchet representations of locally uniform (in the parameter) moderate growth . This introduction of families of Hilbert modules is basically because of the functorial properties of continuous Hilbert families in Appendix $\mathrm{G}$ that I could not prove directly for Fréchet families. The last stage is to start with a holomorphic family of Harish-Chandra modules and use the Hilbert modules corresponding to the resolving $J$-modules and the results of Appendix G to find an open covering of the parameter space and a Hilbert globalization of the family satisfying the smoothability condition over each element of the covering. This yields a family of Fréchet globalizations of local uniform moderate growth on each element of the covering. Proposition F.2 implies that on each of these sets $T$ applied to the family gives a locally continuous family of smooth Fréchet representations. Theorem F.5 implies that this family is locally holomorphic. Our construction of $T$ implies that the corresponding local families of smooth Fréchet representations agree on the intersection of their parameters and thus applying $T$ yields a holomorphic family which completes the proof.

\section{Notation}

Throughout this paper $G$ will denote a real reductive group in the sense of [RRG, 2.1.1] or [BW, 0.3.1] (that is, a finite covering group of an open subgroup of the real points, $\mathbf{G}_{\mathbb{R}}$, of a reductive algebraic group, $\mathbf{G}$, defined over $\mathbb{R}$ ). For the main theorem we will need $G$ to be of inner type (that is $\operatorname{Ad}(G) \subset \operatorname{Ad}\left(\mathbf{G}_{\mathbb{C}}^{o}\right)$ where $\mathbf{G}_{\mathbb{C}}^{o}$ is the identity component of $\mathbf{G}_{\mathbb{C}}$ ) . Let $K$ be a maximal compact subgroup 
of $G$. Throughout the paper, if $H$ is a Lie group over $\mathbb{R}$ then its (real) Lie algebra will be denoted $\mathfrak{h}_{o}$ (i.e. lower case fractur $h$ sub-o) and its complexification denoted $\mathfrak{h}$. Let $\theta$ denote the Cartan involution of $G$ (and of $\mathfrak{g}_{o}$ ) corresponding to $K$. Set $\mathfrak{k}_{o}=\operatorname{Lie}(K), \mathfrak{t}=\mathfrak{t}_{o} \otimes \mathbb{C}$ and $\mathfrak{p}_{o}=\{X \in \mathfrak{g} \mid \theta X=-X\}$. Fix a symmetric $\operatorname{Ad}(G)$ invariant bilinear form, $B$, on $\mathfrak{g}_{o}$ such that $B_{\mid \mathfrak{k}_{o}}$ is negative definite and $B_{\mid \mathfrak{p}_{o}}$ is positive definite. Let $u_{1}, \ldots, u_{n}$ be a basis of $\mathfrak{g}$ and let $v_{1}, \ldots, v_{n}$ be defined by $B\left(u_{i}, v_{j}\right)=\delta_{i j}$ and set $C=\sum_{i=1}^{n} u_{i} v_{i}$ (the corresponding Casimir operator). Let $C_{K}$ be the Casimir operator for $\mathfrak{k}$ corresponding to $B_{\mid \mathfrak{k}}$.

Let, as usual, $\hat{K}$ denote the set of equivalence classes of irreducible continuous representations of $K$. If $V$ is a $K$-module then set $V(\gamma)$ equal to the sum of all irreducible $K$-subrepresentations of $V$ in the class of $\gamma \in \hat{K}$. As is usual, we say that a $K$-module is admissible if

$$
V=\bigoplus_{\gamma \in \hat{K}} V(\gamma)
$$

and $\operatorname{dim} V(\gamma)<\infty$ for all $\gamma \in \hat{K}$.

We denote by $\mathcal{H}(\mathfrak{g}, K)$ the category of Harish-Chandra modules, that is, the finitely generated, admissible, $(\mathfrak{g}, K)$-modules. We also denote by $\mathcal{H} \mathcal{F}(G)$ the category of admissible finitely generated smooth Fréchet representations of moderate growth. This means that an object in $\mathcal{H} \mathcal{F}(G)$ is a pair $(\pi, V)$ with $V$ a Fréchet space and $\pi$ a homomorphism of $G$ into the group of continuous bijections of $V$ such that the following 3 conditions are satisfied

(1) The map $G \times V \rightarrow V$ given by $g, v \mapsto \pi(g) v$ is continuous and is $C^{\infty}$ in $G$.

(2) Let $\|\ldots\|$ be a norm on $G$ (see Appendix C). If $p$ is a continuous seminorm on $V$ then there exists $q$ a continuous seminorm on $V$ and $r$ such that $p(\pi(g) v) \leq$ $\|g\|^{r} q(v)$ for all $v \in V$.

(3) The representation of $\mathfrak{g}, d \pi$, on the $K$-finite vectors of $V, V_{K}$, defines an object in $\mathcal{H}(\mathfrak{g}, K)$.

\section{THE ISOMORPHISM OF CATEGORIES}

One version of the $\mathrm{C}-\mathrm{W}$ Theorem (see [RRG, Theorem 11.6.7]) is

Theorem 2.1. If $(\pi, V),(\mu, W) \in \mathcal{H} \mathcal{F}(G)$ and $L:\left(d \pi, V_{K}\right) \rightarrow\left(d \sigma, W_{K}\right)$ is a morphism in $\mathcal{H}(\mathfrak{g}, K)$ then $L$ extends to a morphism in $\mathcal{H} \mathcal{F}(G)$ with closed image that is a topological summand.

Let $(\pi, V) \in \mathcal{H}(\mathfrak{g}, K)$; then a $K$-invariant Hermitian inner product on $V,\langle\ldots, \ldots\rangle$, will be called $G$-integrable if there exists a strongly continuous action, $\sigma$, of $G$ on the Hilbert space completion of $V, H_{\langle,\rangle}$, relative to $\langle\ldots, \ldots\rangle$, such that the $(\mathfrak{g}, K)$ module of $K$-finite $C^{\infty}$ vectors, $\left(d \sigma,\left(H_{\langle,\rangle}\right)_{K}^{\infty}\right)=(\pi, V)$. The subquotient theorem implies that there exists at least one $G$-integrable inner product on $V$. Let $\mathcal{I}(\pi, V)$ be the set of integrable $K$-invariant inner products on $V$. If $\langle\ldots, \ldots\rangle \in \mathcal{I}(\pi, V)$ then $\left(H_{\langle\ldots, \ldots\rangle}\right)^{\infty} \in \mathcal{H} \mathcal{F}(G)$.

Theorem 2.1 implies that if $\langle\ldots, \ldots\rangle_{i} \in \mathcal{I}(V), i=1,2$ then

$$
\left(H_{\langle\ldots, \ldots\rangle_{1}}\right)^{\infty}=\left(H_{\langle\ldots, \ldots\rangle_{2}}\right)^{\infty} \text {. }
$$

In particular this implies that the norm $v \mapsto\|v\|_{2}$ is continuous on $\left(H_{\langle\ldots, \ldots\rangle_{1}}\right)^{\infty}$. Proposition A.2 implies that there exists constants $B$ and $l$ such that

$$
\|v\|_{2} \leq B\left\|d \sigma_{1}\left(1+C_{K}\right)^{i} v\right\|_{1} .
$$


Note that if $\langle\ldots, \ldots\rangle \in \mathcal{I}(V)$ then the $K$-invariant inner product $\langle v, w\rangle_{1}=$ $\left\langle\pi\left(1+C_{k}\right)^{l} v, w\right\rangle$ is also in $\mathcal{I}(V)$. This allows us to define an inverse to the $K$ finite functor. Set

$$
\bar{V}=\left\{\left\{v_{\gamma}\right\} \in \prod_{\gamma \in \widehat{K}} V(\gamma) \mid \sum_{\gamma \in \widehat{K}}\left\langle v_{\gamma}, v_{\gamma}\right\rangle^{2}<\infty, \forall\langle\ldots, \ldots\rangle \in \mathcal{I}(V)\right\} .
$$

Noting (as above) that this space is equal to $\left(H_{\langle,\rangle}\right)^{\infty}$ for any $\langle\ldots, \ldots\rangle \in \mathcal{I}(V)$ the space $\bar{V}$ endowed with the topology given by the norms $\left\{\|\ldots\|_{\langle\ldots, . .\rangle}\right\}_{\langle\ldots, . .\rangle \in \mathcal{I}(V)}$ is an object in $\mathcal{H} \mathcal{F}(V)$ with $\bar{V}_{K}=V$.

Since $V=\bar{V}_{K}=\oplus_{\gamma \in \hat{K}} V(\gamma) \subset \prod_{\gamma \in \widehat{K}} V(\gamma)$ we have

Theorem 2.2. The functor $V \rightarrow V_{K}$ from $\mathcal{H} \mathcal{F}(G)$ to $\mathcal{H}(\mathfrak{g}, K)$ is an isomorphism of categories with inverse functor $Z \rightarrow \bar{Z}$.

The key aspect of this result for the purposes of this paper is that if $Z \in \mathcal{H F}(G)$ and if for each $\gamma \in \hat{K}$ the $\gamma$-isotypic component of $Z$ is denoted $Z(\gamma)$ then $Z$ is a subspace of $\prod_{\gamma \in \widehat{K}} Z(\gamma)$ as is $Z_{K}=\oplus_{\gamma \in \hat{K}} V(\gamma)$ and $\overline{\left(Z_{K}\right)}=Z$.

In $\mathrm{BK}$ they introduced the notion of "F-space", which is a Fréchet space that has a continuous norm. Using the argument in the proof of Lemma 2.A.2.1 in [RRG one sees that a strongly continuous representation of a real reductive group on an F-space is of moderate growth. Also the results above imply that if $V$ is an admissible, smooth Fréchet module of moderate growth then $V$ is an $\mathrm{F}$-space.

The rest of this paper will be devoted to the study of the dependence of this functor on parameters. For this we will use a class of universal modules with remarkable properties related to ones in [RRG, Section 11.3] and in [HOW].

\section{The subalgebra D of $Z(\mathfrak{g})$}

Throughout the rest of this paper $G$ will be a real reductive group of inner type (see Section 1). We keep the notation of the previous section. Also fix a symmetric $A d(G)$-invariant bilinear form on $\mathfrak{g}_{o}$ such that if $\theta$ is the Cartan involution relative to $K$ then $\langle X, Y\rangle=-B(\theta X, Y)$ defines an inner product on $\mathfrak{g}_{o}$. Let $p$ be the projection of $\mathfrak{g}$ onto $\mathfrak{p}=\mathfrak{p}_{o} \otimes \mathbb{C}$ corresponding to $\mathfrak{g}_{o}=\mathfrak{k}_{o} \oplus \mathfrak{p}_{o}$. Extend $p$ to a homomorphism of $S(\mathfrak{g})$ onto $S(\mathfrak{p})$. Then $p$ is the projection corresponding to

$$
S(\mathfrak{g})=S(\mathfrak{p}) \oplus S(\mathfrak{g}) \mathfrak{k} .
$$

In [HOW, Theorem 2.3] we found homogeneous elements $w_{1}, \ldots, w_{l}$ of $S(\mathfrak{g})^{G}$, with $w_{1}=C$, satisfying the following two properties:

(1) $p\left(w_{1}\right), \ldots, p\left(w_{l}\right)$ are algebraically independent.

(2) There exists a finite dimensional homogeneous subspace $E$ of $S(\mathfrak{p})^{K}$ such that the map $\mathbb{C}\left[p\left(w_{1}\right), \ldots, p\left(w_{l}\right)\right] \otimes E \rightarrow S(\mathfrak{p})^{K}$ given by multiplication is an isomorphism.

In $[\mathrm{H}]$ Helgason proved that the only simple Lie algebras over $\mathbb{R}$ for which $E \neq \mathbb{C} 1$ are of type E. Here is the list and the dimensions of the corresponding spaces $E$ (cf. Proposition 2.1 in $[\mathrm{HOW}]$ ): the two real rank two real forms of $\mathrm{E}_{6}$ with $\operatorname{dim} E=2$, the real rank 3 real form of $E_{7}$ where $\operatorname{dim} E=2$ and the real rank 4 real form of $\mathrm{E}_{8}$ where $\operatorname{dim} E=4$. 
Let $\mathcal{H}$ denote the space of harmonic elements of $S(\mathfrak{p})$, that is, the orthogonal complement to the ideal $S(\mathfrak{p})(S(\mathfrak{p}) \mathfrak{p})^{K}$ in $S(\mathfrak{p})$ relative to the Hermitian extension of $B_{\mid \mathfrak{p}_{o}}$. Then the Kostant-Rallis theorem [KR] implies that the map

$$
\mathcal{H} \otimes S(\mathfrak{p})^{K} \rightarrow S(\mathfrak{p})
$$

given by multiplication is a linear bijection. This and (2) easily imply

Lemma 3.1. The map

$$
\mathcal{H} \otimes E \otimes \mathbb{C}\left[w_{1}, \ldots, w_{l}\right] \otimes S(\mathfrak{k}) \rightarrow S(\mathfrak{g})
$$

given by multiplication is a linear bijection.

Let $\mathfrak{a}_{o}$ be a maximal abelian subspace of $\mathfrak{p}_{o}$ and let (as usual)

$$
W=W(\mathfrak{a})=\left\{s \in G L(\mathfrak{a}) \mid s=A d(k)_{\mid \mathfrak{a}}, k \in K\right\} .
$$

Let $h \in \mathfrak{a}_{o}$ be such that $\mathfrak{a}_{o}=\left\{X \in \mathfrak{p}_{o} \mid[h, X]=0\right\}$. If $\lambda \in \mathbb{R}$ then set $\mathfrak{g}_{o}^{\lambda}=\{X \in$ $\left.\mathfrak{g}_{o} \mid[h, X]=\lambda X\right\}$. Set $\mathfrak{n}_{o}=\oplus_{\lambda>0} \mathfrak{g}_{o}^{\lambda}$ and $\overline{\mathfrak{n}}_{o}=\theta \mathfrak{n}_{o}=\oplus_{\lambda>0} \mathfrak{g}_{o}^{-\lambda}$. Then

$$
\mathfrak{p}=p(\mathfrak{n}) \oplus \mathfrak{a}
$$

and $p(\mathfrak{n})$ is the orthogonal complement to $\mathfrak{a}$ in $\mathfrak{p}$ relative to $B$. Let $q$ be the projection of $\mathfrak{p}$ onto $\mathfrak{a}$ corresponding to this decomposition. Then the Chevalley restriction theorem implies that

$$
q: S(\mathfrak{p})^{K} \rightarrow S(\mathfrak{a})^{W}
$$

is an isomorphism of algebras. Also, as above, if $H$ is the orthogonal complement to $(S(\mathfrak{a}) \mathfrak{a})^{W} S(\mathfrak{a})$ in $S(\mathfrak{a})$. Then the map

$$
S(\mathfrak{a})^{W} \otimes H \rightarrow S(\mathfrak{a})
$$

given by multiplication is a linear bijection. Putting these observations together the map

$$
S(\mathfrak{n}) \otimes S(\mathfrak{a})^{W} \otimes H \otimes S(\mathfrak{k}) \rightarrow S(\mathfrak{g})
$$

given by multiplication is a linear bijection. We also note that the map

$$
\mathbb{C}\left[w_{1}, \ldots, w_{l}\right] \otimes E \rightarrow S(\mathfrak{a})^{W}
$$

given by

$$
w \otimes e \mapsto q(p(w)) q(e)
$$

is a linear bijection. This in turn implies

Lemma 3.2. The map

$$
S(\mathfrak{n}) \otimes \mathbb{C}\left[w_{1}, \ldots, w_{l}\right] \otimes E \otimes H \otimes S(\mathfrak{k}) \rightarrow S(\mathfrak{g})
$$

given by multiplication is a linear bijection.

Let symm denote the symmetrization map from $S(\mathfrak{g})$ to $U(\mathfrak{g})$; then symm is a linear bijection and symm $\circ A d(g)=A d(g) \circ$ symm if $g \in G$. Let $Z(\mathfrak{g})=U(\mathfrak{g})^{G}$ denote the center of $U(\mathfrak{g})$. Set $z_{i}=\operatorname{symm}\left(w_{i}\right)$ and

$$
\mathbf{D}=\mathbb{C}\left[z_{1}, \ldots, z_{l}\right] \text {. }
$$

Note that if $S_{j}(\mathfrak{g})=\sum_{k \leq j} S^{j}(\mathfrak{g})$ and if $U^{j}(\mathfrak{g}) \subset U^{j+1}(\mathfrak{g})$ is the standard filtration of $U\left(\mathfrak{g}_{\mathbb{C}}\right)$ then

$$
\operatorname{symm}\left(S_{j}(\mathfrak{g})\right)=U^{j}(\mathfrak{g}) .
$$

The above and standard arguments ([HOW, Theorem 2.5 and Lemma 5.2]) imply 
Theorem 3.3. Let the notation be as above. Then

(1) The map

$$
\mathcal{H} \otimes E \otimes \mathbf{D} \otimes U(\mathfrak{k}) \rightarrow U(\mathfrak{g})
$$

given by

$$
h \otimes e \otimes D \otimes k \mapsto \operatorname{symm}(h) \operatorname{symm}(e) D k
$$

is a linear bijection.

(2) The map

$$
U(\mathfrak{n}) \otimes E \otimes H \otimes \mathbf{D} \otimes \mathbf{U}(\mathfrak{k}) \rightarrow U(\mathfrak{g})
$$

given by

$$
n \otimes e \otimes h \otimes D \otimes k \mapsto n \operatorname{symm}(e) \operatorname{symm}(h) D k
$$

is a linear bijection.

\section{A Class of admissible finitely generated $(\mathfrak{g}, K)$-Modules}

Retain the notation in the preceding section. Note that Theorem 3.3 implies that the subalgebra $\mathbf{D} U(\mathfrak{k})$ of $U(\mathfrak{g})$ is isomorphic with the tensor product algebra $\mathbf{D} \otimes U(\mathfrak{k})$ and that $U(\mathfrak{g})$ is free as a right $\mathbf{D} U(\mathfrak{k})$-module under multiplication. If $R$ is a $\mathbf{D} U(\mathfrak{k})$-module then set

$$
J(R)=U(\mathfrak{g}) \otimes_{\mathbf{D} U(\mathfrak{k})} R .
$$

Recall that $\mathcal{H}(\mathfrak{g}, K)$ denotes the Harish-Chandra category of admissible finitely generated $(\mathfrak{g}, K)$-modules. Let $R$ be a finite dimensional continuous $K$-module that is also a $\mathbf{D}$-module such that the actions commute; then $K$ acts on $J(R)$ as follows:

$$
k \cdot(g \otimes r)=A d(k) g \otimes k r, k \in K, g \in U(\mathfrak{g}), r \in R .
$$

As a $K$-module

$$
J(R) \cong \mathcal{H} \otimes E \otimes R
$$

with $K$ acting trivially on $E$. Note that $J(R) \in \mathcal{H}(\mathfrak{g}, K)$ since the multiplicities of $K$-types in $\mathcal{H}$ are finite and $J(R)$ is clearly finitely generated as a $U(\mathfrak{g})$-module. Let $W(\mathbf{D}, K)$ be the category of finite dimensional $(\mathbf{D}, K)$-modules with $K$ acting continuously and such that the action of $\mathbf{D}$ and $K$ commute.

Lemma 4.1. $R \rightarrow J(R)$ defines an exact faithful functor from the category $W(\mathbf{D}, K)$ to $\mathcal{H}(\mathfrak{g}, K)$.

Proof. This is a consequence of the freeness of $U(\mathfrak{g})$ as a module for $\mathbf{D} U(\mathfrak{k})$ under right multiplication.

If $V \in \mathcal{H}(\mathfrak{g}, K)$ then $V(\gamma)$ is invariant under the action of $Z(\mathfrak{g})$ hence under the action of $\mathbf{D}$.

By definition, if $V \in \mathcal{H}(\mathfrak{g}, K)$ there is a finite subset $F \subset \hat{K}$ such that

$$
U(\mathfrak{g}) \sum_{\gamma \in F} V(\gamma)
$$

Set $R=\sum_{\gamma \in F} V(\gamma) \in W(\mathbf{D}, K)$. One has a canonical $(\mathfrak{g}, K)$-module surjection $J(R) \rightarrow V$ given by $g \otimes r \mapsto g r$. A submodule of an element of $\mathcal{H}(\mathfrak{g}, K)$ is in $\mathcal{H}(\mathfrak{g}, K)$ so

Proposition 4.2. If $V \in \mathcal{H}(\mathfrak{g}, K)$ then there exists a sequence of elements $R_{j} \in$ $W(\mathfrak{g}, K)$ and an exact sequence in $\mathcal{H}(\mathfrak{g}, K)$

$$
\cdots \rightarrow J\left(R_{k}\right) \rightarrow \ldots \rightarrow J\left(R_{2}\right) \rightarrow J\left(R_{1}\right) \rightarrow J\left(R_{0}\right) \rightarrow V \rightarrow 0 .
$$


Notice that this exact sequence is a free resolution of $V$ as a $U(\mathfrak{n})$-module.

Let $\beta: \mathbf{D} \rightarrow \mathbb{C}$ be an algebra homomorphism. Let $\mathcal{H}(\mathfrak{g}, K)_{\beta}$ be the full subcategory of $\mathcal{H}(\mathfrak{g}, K)$ consisting of modules $V$ such that if $z \in \mathbf{D}$ then it acts by $\beta(z) I$. The next result is an aside that will not be used in the rest of this paper and is a simple consequence of the definition of projective object.

Lemma 4.3. Let $R$ be a finite dimensional continuous $K$-module and let $\mathbf{D}$ act on $F$ by $z \mapsto \beta(z) I$ with $\beta \in \operatorname{Hom}_{\mathbf{D}}(\mathbf{D}, \mathbb{C})$ yielding an object $R \in W(\mathbf{D}, K)$. Then $J(R)$ is projective in $\mathcal{H}(\mathfrak{g}, K)_{\beta}$.

\section{The objects in $W(\mathbf{D}, K)$}

If $R \in W(\mathbf{D}, K)$ then $R$ has a $K$-isotypic decomposition $R=\oplus_{\gamma \in \hat{K}} R(\gamma)$. Only a finite number of the $R(\gamma)$ are non-zero. If $D \in \mathbf{D}$ then $D R(\gamma) \subset R(\gamma)$ for all $\gamma \in \hat{K}$. If $\chi: \mathbf{D} \rightarrow \mathbb{C}$ is an algebra homomorphism then we set $R_{\chi}=\{v \in$ $R \mid(D-\chi(D))^{k} v=0$, for some $\left.k>0\right\}$. Then setting $\operatorname{ch}(\mathbf{D})$ equal to the set of all algebra homomorphisms of $\mathbf{D}$ to $\mathbb{C}$ we have the decomposition

$$
R=\bigoplus_{\gamma \in \hat{K}, \chi \in \operatorname{ch}(\mathbf{D})} R_{\chi}(\gamma)
$$

Fix a $K$-module $\left(\tau_{\gamma}, F_{\gamma}\right) \in \gamma$. Then $R_{\chi}(\gamma)$ is isomorphic with

$$
\operatorname{Hom}_{K}\left(V_{\gamma}, R_{\chi}\right) \otimes F_{\gamma}
$$

with $K$ acting on $F_{\gamma}$ and $\mathbf{D}$ acting on $\operatorname{Hom}_{K}\left(V_{\gamma}, R\right)$.

If $R$ is an irreducible object in $W(\mathbf{D}, K)$ then Schur's lemma implies that $\mathbf{D}$ acts by a homomorphism $\chi: \mathbf{D} \rightarrow \mathbb{C}$ and $R$ is irreducible as a $K$-module. Set $V_{\gamma, \chi}$, equal to the module with $\mathbf{D}$ acting by $\chi$ and $K$ acting by an element of $\gamma$.

Let $\chi$ be such a homomorphism then $\chi\left(z_{i}\right)=\lambda_{i} \in \mathbb{C}$. Thus it is parametrized by $\left(\lambda_{1} \ldots, \lambda_{l}\right) \in \mathbb{C}^{l}$. We will use the notation $\beta_{\lambda}$ for the homomorphism such that $\beta_{\lambda}\left(z_{i}\right)=\lambda_{i}$.

Definition 5.1. Let $X$ be an analytic manifold. An analytic family in $W(\mathbf{D}, K)$ over $X$ is a pair $(\mu, V)$ of a finite dimensional continuous $K$-module, $V$, and a $\mu: X \times \mathbf{D} \rightarrow \operatorname{End}(V)$ such that $D \mapsto \mu(x, D)$ is a representation of $\mathbf{D}$ on $V$ and $x \mapsto \mu(x, D)$ is analytic for all $D \in \mathbf{D}$.

\section{Parabolically induced families}

Let $A$ and $N$ be the connected subgroups of $G$ with $\operatorname{Lie}(A)=\mathfrak{a}_{o}$ and $\operatorname{Lie}(N)=$ $\mathfrak{n}_{o}$. Let $M$ be the centralizer of $\mathfrak{a}$ in $K$. Set $Q=M A N$; then $Q$ is a minimal parabolic subgroup of $G$.

Definition 6.1. An analytic family of finite dimensional $Q$-modules over a real analytic manifold $X$ is a pair $(\sigma, S)$ with $S$ a finite dimensional continuous $M$ module and a real analytic map $\sigma: X \times Q \rightarrow G L(S)$ such that $x \mapsto \sigma(x, q)$ is analytic and $\sigma(x, \cdot)=\sigma_{x}$ is a representation of $Q$.

Let $(\sigma, S)$ be a continuous finite dimensional representation of $Q$. Set $I^{\infty}\left(\sigma_{\mid M}\right)$ equal to the space of all smooth functions $f: K \rightarrow S$ satisfying $f(m k)=\sigma(m) f(k)$ for $m \in M, k \in K$. Define an action $\pi_{\sigma}$ of $G$ on $I^{\infty}\left(\sigma_{\mid M}\right)$ as follows: if $f \in I^{\infty}\left(\sigma_{\mid M}\right)$ 
then extend $f$ to $G$ by $f_{\sigma}(q k)=\sigma(q) f(k)$; then, since $K \cap Q=M$ and $Q K=G$, $f_{\sigma}$ is $C^{\infty}$ on $G$. Set $\pi_{\sigma}(g) f(k)=f_{\sigma}(k g)$. Also set

$$
\pi_{\sigma}(Y) f(k)=\frac{d}{d t} f_{\sigma}(k \exp t Y)_{\mid t=0}
$$

for $Y \in \mathfrak{g}_{o}$ and $k \in K, f \in I^{\infty}\left(\sigma_{\mid M}\right)$. Let $I\left(\sigma_{\mid M}\right)$ be the space of all right $K$ finite elements of $I^{\infty}\left(\sigma_{\mid M}\right)$.

Put an $M$-invariant inner product, $\langle\ldots, \ldots\rangle$ on $S$. If $f, h \in I^{\infty}\left(\sigma_{\mid M}\right)$ then set

$$
(f, h)=\int_{K}\langle f(k), h(k)\rangle d k
$$

with $d k$ normalized invariant measure on $K$. The following observation is standard.

Proposition 6.2. Let $(\sigma, S)$ be an analytic family of finite dimensional representations of $Q$ over the analytic manifold $X$. Set $\lambda(x, y)=\pi_{\sigma_{x}}(y)$ for $x \in X, y \in U\left(\mathfrak{g}_{\mathbb{C}}\right)$. If $\mu$ is the common value of $\left.\sigma_{x}\right|_{M}$, then $(\lambda, I(\mu))$ is an analytic family (see Appendix D) of objects in $\mathcal{H}(\mathfrak{g}, K)$ over $X$.

Proposition 6.3. Let $(\sigma, S)$ be an analytic family of $Q$-modules based on $Z$. Set $\sigma(m)$ equal to the common value of $\sigma_{z}(m)$ for $m \in M$ and $H$ equal to the unitarily induced representation of $\sigma$ from $M$ to $K$. Then $z \rightarrow\left(\pi_{\sigma_{z}}, H\right)$ is a continuous family of Hilbert representations over $Z$ (see Definition B.1) that is smoothable in the sense of Definition F.3.

Proof. Let $f \in I^{\infty}(\sigma)$ that is

$$
f(m k)=\sigma(m) f(k), m \in M, k \in K .
$$

Recall that

$$
f_{\sigma_{x}}(g)=f_{\sigma_{x}}(n a m k)=\sigma_{x}(\text { nam }) f(k)
$$

for $g=n a m k, n \in N, a \in A, m \in M, k \in K$. Let $\left\{n_{1} . n_{2}, \ldots\right\},\left\{a_{1}, a_{2}, \ldots\right\}$ be respectively bases of $U(\mathfrak{n})$ and $U(\mathfrak{a})$ compatible with the standard filtration of $U(\mathfrak{g})$. Let $Y_{1}, \ldots, Y_{n}$ be a basis of $\mathfrak{k}_{o}$ such that $B\left(Y_{i}, Y_{j}\right)=-\delta_{i j}$. The monomials $Y^{I}=Y_{1}^{i_{1}} \cdots Y_{n}^{i_{n}}$ form a basis of $U(\mathfrak{k})$. If $u \in U(\mathfrak{g})$ and if $f \in H^{\infty}$ then

$$
d \pi_{\sigma_{z}}(u) f(k)=L\left(A d\left(k^{-1}\right) u^{T}\right) f_{\sigma_{z}}(k)
$$

with $L$ the left action of $U(\mathfrak{g})$ on $C^{\infty}(G, S)$ and $u \mapsto u^{T}$ is the standard antiinvolution of $U(\mathfrak{g})$ defined by $1 \mapsto 1$ and $X \mapsto-X$ for $X \in \mathfrak{g}$. Also

$$
A d\left(k^{-1}\right) u^{T}=\sum_{i, j, I} a_{i, j, I}(k) n_{i} a_{j} Y^{I}
$$

finite sum with the set of indices such that $a_{i, j, I}(k) \neq 0$ depending only on the level of $u$ in the standard filtration of $U(\mathfrak{g})$. Thus

Writing

$$
\begin{gathered}
d \pi_{\sigma_{z}}(u) f(k)=\sum a_{i, j, I}(k) d \sigma_{z}\left(n_{i} a_{j}\right)\left(L\left(Y^{I}\right) f\right)(k) \\
=\sum a_{i, j, I}(k) d \sigma_{z}\left(n_{i} a_{j}\right)\left(A d(k)^{-1}\left(Y^{I}\right)^{T} f\right)(k) .
\end{gathered}
$$

we have

$$
A d(k)^{-1}\left(Y^{I}\right)^{T}=\sum_{|J| \leq|I|} b_{J, I}(k) Y^{J}
$$

$$
d \pi_{\sigma_{z}}(u) f(k)=\sum_{i, j, I, J} b_{J, I}(k) a_{i, j, I}(k) d \sigma_{z}\left(n_{i} a_{j}\right) Y^{J} f(k) .
$$


Since the sum is finite, all of the indices are bounded. Let $\omega$ be a compact subset of $Z$ then for each fixed $J$

$$
\sum_{i, j, I}\left|b_{J, I}(k) a_{i, j, I}(k)\right|\left\|d \sigma_{z}\left(n_{i} a_{j}\right)\right\| \leq C_{u, \omega, J}^{1}, k \in K, z \in \omega .
$$

Thus

$$
\begin{aligned}
\left\|d \pi_{\sigma_{z}}(u) f\right\|^{2} & \leq \sum C_{u, \omega, L}^{1} C_{u, \omega, J}^{1}\left\langle Y^{L} f, Y^{J} f\right\rangle \\
\leq & C_{u, \omega}\left\|\left(1+C_{K}\right)^{l} f\right\|^{2}
\end{aligned}
$$

with $l$ the maximum of the $|J|$ for the multi-indices that appear in the formulas above.

\section{Analytic families of $J$-modules}

Notation will be as in the previous section. Throughout this section analytic will mean complex analytic in the context of a complex analytic manifold and real analytic in the context of a real analytic manifold.

Proposition 7.1. Let $X$ be a real analytic or complex manifold. Let $(\lambda, R)$ be a family of objects in $W(\mathbf{D}, K)$ over $X$ and define $R_{x} \in W(\mathbf{D}, K)$ to be the module with action $\lambda(x, \cdot)$. Let

$$
V=\mathcal{H} \otimes E \otimes R
$$

( $K$ acts by the tensor product action with its action on $E$ trivial) and let $T_{x}: V \rightarrow$ $J\left(R_{x}\right)$ be given by $T_{x}(h \otimes e \otimes r)=\alpha_{x}(\operatorname{symm}(h) e)(1 \otimes r)$ with $\alpha_{x}$ the action of $U\left(\mathfrak{g}_{\mathbb{C}}\right)$ on $J\left(R_{x}\right)$. If $\lambda(x, y)=T_{x}^{-1} \alpha_{x}(y) T_{x}$ then $(\lambda, V)$ is an analytic family of objects in $\mathcal{H}(\mathfrak{g}, K)$ based on $X$.

Proof. Let $\left\{h_{i}\right\}$ be a basis of $\mathcal{H}$ such that for each $i$ there exists $\gamma \in \hat{K}$ such that $h_{i} \in \mathcal{H}(\gamma)$, let $\left\{e_{j}\right\}$ be a basis of $E$, let $\left\{r_{m}\right\}$ be a basis of $R$ and let $\left\{Y_{1}, \ldots, Y_{n}\right\}$ be a basis of $\mathfrak{k}$. If $y \in U\left(\mathfrak{g}_{\mathbb{C}}\right)$ then

$$
y \operatorname{symm}\left(h_{i}\right) e_{j} z^{L} Y^{J}=\sum_{i_{1}, j_{1}, J_{1}, L_{1}} b_{i_{1} j_{1} L_{1} J_{1}, i j L K}(y) \operatorname{symm}\left(h_{i_{1}}\right) e_{j_{1}} z^{L_{1}} Y^{J_{1}} .
$$

Thus

$$
T_{x}^{-1} \alpha_{x}(y) T_{x}\left(h_{i} \otimes e_{j} \otimes r_{k}\right)=\sum b_{i_{1} j_{1} L_{1} J_{1}, i j 00}(y) h_{i_{1}} \otimes e_{j_{1}} \otimes\left(\lambda_{x}\left(z^{L_{1}}\right) Y^{J_{1}} r_{k}\right) .
$$

The proposition follows.

Theorem 3.3 implies

Lemma 7.2. Let $R \in W(\mathbf{D}, K)$; then

$$
J(R) / \mathfrak{n}^{k+1} J(R) \cong\left(U(\mathfrak{n}) / \mathfrak{n}^{k+1} U(\mathfrak{n})\right) \otimes E \otimes H \otimes R_{\mid M}
$$

as an $(\mathfrak{n}, M)$-module with $\mathfrak{n}$ acting by left multiplication on $U(\mathfrak{n}) / \mathfrak{n}^{k+1} U(\mathfrak{n})$ and trivially $R, M$ acting trivially on $E \otimes H$, and $m \in M$ acting by $A d(m)$ on $U(\mathfrak{n}) / \mathfrak{n}^{k+1} U(\mathfrak{n})$ and by the restriction of the action of $K$ on $R$.

Let $(\mu, R)$ be an analytic family of objects in $W(\mathbf{D}, K)$ over $X$. Let $R_{x}, x \in X$ be the object in $W(\mathbf{D}, K)$ with $K$ acting only on $R$ and $\mathbf{D}$ acting by $\mu_{x}=\mu(x, \cdot)$. 
Proposition 7.3. Let $p_{x, k}: J\left(R_{x}\right) \rightarrow\left(U(\mathfrak{n}) / \mathfrak{n}^{k+1} U(\mathfrak{n})\right) \otimes E \otimes H \otimes R_{\mid M}$ be given by the projection of $J\left(R_{x}\right)$ onto $J(R) / \mathfrak{n}^{k+1} J(R)$ composed with the canonical isomorphism of $J(R) / \mathfrak{n}^{k+1} J(R)$ with $\left(U(\mathfrak{n}) / \mathfrak{n}^{k+1} U(\mathfrak{n})\right) \otimes E \otimes H \otimes R$. If $v \in J\left(R_{x}\right)$ and $u \in U\left(\mathfrak{g}_{\mathbb{C}}\right)$ then the map

$$
x \mapsto p_{x, k}(u v)
$$

is analytic from $X$ to $\left(U(\mathfrak{n}) / \mathfrak{n}^{k+1} U(\mathfrak{n})\right) \otimes E \otimes H \otimes R_{\mid M}$. In particular, if $p_{k}$ is the canonical projection of $U(\mathfrak{n}) \otimes E \otimes H \otimes R_{\mid M}$ onto

$$
\left(U(\mathfrak{n}) / \mathfrak{n}^{k+1} U(\mathfrak{n})\right) \otimes E \otimes H \otimes R_{\mid M},
$$

then define $\sigma_{k, x}(q) p_{k}(v)=p_{x, k}(q v), q \in \operatorname{Lie}(Q)$ since $\left(U(\mathfrak{n}) / \mathfrak{n}^{k+1} U(\mathfrak{n})\right) \otimes E \otimes H \otimes R$ is finite dimensional and $A N$ is simply connected; this action integrates to a $Q$ module structure so

$$
\left(\sigma_{k},\left(U(\mathfrak{n}) / \mathfrak{n}^{k+1} U(\mathfrak{n})\right) \otimes E \otimes H \otimes R\right)
$$

is an analytic family of finite dimensional $Q$-modules.

Proof. Let $x_{1}, x_{2}, \ldots, x_{r}$ be a linearly independent set in $U(\mathfrak{n})$ that projects to a basis in $U(\mathfrak{n}) / \mathfrak{n}^{k+1} U(\mathfrak{n})$ and let $x_{r+1}, \ldots$ be a basis of $\mathfrak{n}^{k+1} U(\mathfrak{n})$. Theorem 3.3 implies that if $Y_{1}, \ldots, Y_{n}$ is a basis of $\mathfrak{k}$ and $h_{1}, \ldots, h_{r}$ is a basis for $\operatorname{symm}(E) \operatorname{symm}(H)$ then if $J$ is a multi-index of size $n$ and $I$ is a multi-index of size then the set of elements

$$
x_{l} z^{I} h_{m} Y^{J}
$$

is a basis of $U\left(\mathfrak{g}_{\mathbb{C}}\right)\left(z^{I}=z_{1}^{i_{1}} \cdots z_{l}^{i_{l}}\right)$. This implies that if $u \in U\left(\mathfrak{g}_{\mathbb{C}}\right)$ then

$$
u x_{s} z^{I} h_{t} Y^{J}=\sum_{s_{1}, I_{1}, t_{1}, J_{1}} a_{s_{1} J_{1} t_{1} L_{1}, s I t J}(u) X^{J_{1}} z^{L_{1}} h_{i_{1}} Y^{J_{1}} .
$$

If we take a basis $v_{1}, \ldots, v_{d}$ of $R$ then the elements $X^{J} h_{i} \otimes v_{j}$ form a basis of $J\left(V_{x}\right)$. Thus, if $u \in U\left(\mathfrak{g}_{\mathbb{C}}\right)$ then

$$
\begin{gathered}
u x_{s} h_{t} \otimes v_{j}=\sum a_{s_{1} I_{1} t_{1} J_{1}, s, 0, t, 0}(u) x_{s_{1}} z^{I_{1}} h_{t_{1}} Y^{J_{1}} \otimes v_{j u} \\
=\sum a_{s_{1} I_{1} t_{1} J_{1}, s, 0, t, 0}(u) x_{s} h_{t} \otimes \mu_{x}\left(z^{I_{1}}\right) Y^{J_{1}} v_{j} .
\end{gathered}
$$

Now apply $p_{k, x}$ getting the image of

$$
\sum_{s_{1} \leq r} a_{s_{1} I_{1} t_{1} J_{1}, s, 0, t, 0}(u) x_{s} h_{t} \otimes\left(\mu_{x}\left(z^{I_{1}}\right) Y^{J_{1}} v_{j}\right) .
$$

The proposition follows from this formula.

Let (as above) $p_{s}$ denote the natural surjection

$$
p_{s}: J(R) \rightarrow J(R) / \mathfrak{n}^{s+1} J(R) .
$$

Let $\sigma_{s, R}$ denote the family $\sigma_{s}$ defined above. If $k \in K, v \in J(R)$, define $S_{s, R}(v)(k)=$ $p_{s, R}(k v)$; then $S_{s, R}(v) \in I\left(\left.\sigma_{s, R}\right|_{M}\right)$ and it is easily seen that $S_{s, R} \in \operatorname{Hom}_{\mathcal{H}(\mathfrak{g}, K)}(J(R)$, $\left(\pi_{\sigma_{s, R}}, I\left(\left.\sigma_{s, R}\right|_{M}\right)\right)$. Combining the above results we have

Theorem 7.4. Let $(\mu, R)$ be an analytic (resp. continuous) family in $W(\mathbf{D}, K)$ based on the manifold $X$. Let $(\lambda, V)$ be the analytic family (as in Proposition 7.1) corresponding to $x \rightarrow J\left(\left(\mu_{x}, R\right)\right)$. Then recalling that $V=\mathcal{H} \otimes E \otimes R$ define $T_{s}(x)(h \otimes e \otimes r)=S_{s}, R_{x}(\operatorname{symm}(h) e \otimes r)$. Then $T_{s}$ defines a homomorphism of the analytic family $(\lambda, V)$ to $\left(\xi, I\left(\sigma_{s, R_{x}},\left.\right|_{M}\right)\right)$ (in the sense of Definition D.3) with $\xi(x, y)=\pi_{\sigma_{s, R_{x}}}(y)$ and $\sigma_{s, R_{x}}$ is defined as above. 
We will use the notation $J(R)$ for the analytic family associated with $x \rightarrow$ $J\left(\left(\mu_{x}, R\right)\right)$.

\section{Imbeddings of $J$-MOdules AND THEIR HilberT FAMily COMPLETIONS}

Let $X$ be a connected real or complex analytic manifold and let $(\mu, R)$ be an analytic family of objects in $W(\mathbf{D}, K)$ based on $X$. We maintain the notation of the previous section. The purpose of this section is to prove

Theorem 8.1. Let the representation of $Q, \sigma_{k, x}$, on

$$
W_{k}=\left(U(\mathfrak{n}) / \mathfrak{n}^{k+1} U(\mathfrak{n})\right) \otimes E \otimes H \otimes R_{\mid M}
$$

be as in Proposition 7.3 and let $T_{k}(x)$ be the analytic family as in Theorem 7.4 . If $\omega$ is a compact subset of $X$ then there exists $k_{\omega}$ such that if $x \in \omega$ then $T_{k}(x)$ is injective for $k \geq k_{\omega}$.

Proof. This is a slight extension of a result in HOW. Given $k$, then $\left(\sigma_{k, x}, W_{k}\right)$ as a composition series $W_{k, x}=W_{k, x}^{1} \supset W_{k, x}^{2} \supset \cdots \supset W_{k, x}^{r} \supset W_{k, x}^{r+1}=\{0\}$ and each $W_{k, x}^{i} / W_{k, x}^{i+1}$ is isomorphic with the representation $\left(\lambda_{j, \nu_{j}}, H_{\lambda_{j}}\right)$ with $\left(\lambda_{j}, H_{j}\right)$ an irreducible representation of $M$ and $\nu_{j} \in \mathfrak{a}_{\mathbb{C}}^{*}$ and $\lambda_{j, \nu}(\operatorname{man})=a^{\nu+\rho} \lambda_{j}(m)$ with $m \in M, a \in A$ and $n \in N$. Also note that there is a natural $Q$-module exact sequence

$$
0 \rightarrow\left(\mathfrak{n}^{k+1} U(\mathfrak{n}) / \mathfrak{n}^{k+2} U(\mathfrak{n})\right) \otimes E \otimes H \otimes R_{\mid M} \rightarrow W_{k+1, x} \rightarrow W_{k, x} \rightarrow 0 .
$$

We may assume that the composition series is consistent with this exact sequence. This implies that the $\nu_{j}$ that appear in $W_{k} / W_{k+1}$ are of the form $\mu+\alpha_{1}+\cdots+\alpha_{k+1}$ with $\alpha_{i}$ a restricted positive root (i.e. a weight of $\mathfrak{a}$ on $\mathfrak{n}$ ).

Now consider the corresponding exact sequence of $(\mathfrak{g}, K)$-modules.

$$
(*) 0 \rightarrow I\left(\eta_{k, x}\right) \rightarrow I\left(\sigma_{k+1, x}\right) \rightarrow I\left(\sigma_{k, x}\right) \rightarrow 0 .
$$

The $(\mathfrak{g}, K)$-modules $I\left(\sigma_{\nu}\right)$ with $\sigma$ an irreducible representation of $M$ with HarishChandra parameter $\Lambda_{\sigma}$ (for $\left.\operatorname{Lie}(M)_{\mathbb{C}}\right)$ and $\nu \in \mathfrak{a}_{\mathbb{C}}^{*}$ have infinitesimal character with Harish-Chandra parameter $\Lambda_{\sigma}+\nu$. We are finally ready to prove the theorem with notation as in Appendix E.

Let $C_{\omega}$ be the compact set $\cup_{x \in \omega} \operatorname{ch}\left(J\left(R_{x}\right)\right)$. Let $C_{\omega}=\cup_{i=1}^{k_{\omega}}\left(\Lambda_{i}+D_{i}\right)$ with $D_{i}$ compact in $\mathfrak{a}_{\mathbb{C}}^{*}$ and $k_{\omega}<\infty$. Assume that the result is false for $\omega$. Then for each $j$ there exists $k \geq j$ and $x$ such that $\operatorname{ker} T_{k}(x) \neq 0$ but $\operatorname{ker} T_{k+1}(x)=0$. Label the Harish -Chandra parameters that appear in $I\left(\sigma_{o, x}\right), \Lambda_{1}+\nu_{1}, \ldots, \Lambda_{s}+\nu_{s}$ with $\Lambda_{i} \in \operatorname{Lie}(T)^{*}$ and $\nu_{i} \in \mathfrak{a}_{\mathbb{C}}^{*}$ (recall that we have fixed a maximal torus of $M)$. The above observations imply that $\operatorname{ch}\left(J\left(R_{x}\right)\right)$ contains an element of the form $\Lambda+\nu_{i_{k}}+\beta_{k}$ with $\beta_{k}$ a sum of $k$ positive roots, $\Lambda \in \operatorname{Lie}(T)^{*}$ and $1 \leq i_{k} \leq s$. We now have our contradiction $\nu_{i_{k}}+\beta_{k} \in \cup D_{i}$ which is compact. But the set of $\nu_{i_{k}}+\beta_{k}$ is unbounded.

Theorem 8.2. Let $U \subset Z$ be open with compact closure. There exists a continuous family $(\pi, H)$ of Hilbert representations of $G$ (see Definition B.1) based on $U$ such that the continuous family of $(\mathfrak{g}, K)$-modules $\left(d \pi, H_{K}^{\infty}\right)$ is isomorphic with the analytic family $z \mapsto J\left(L_{z}\right)$ of objects in $\mathcal{H}(\mathfrak{g}, K)$ based on $U$ (thought of as a continuous family). Furthermore, the family $(\pi, H)$ is smoothable (see Definition F.3). 
Proof. Let $\gamma \in \hat{K}$ then Theorem 3.3) (2) implies

$$
\operatorname{dim} J\left(L_{z}\right)(\gamma)=\operatorname{dim} E \operatorname{dim} \gamma \operatorname{dim} \operatorname{Hom}_{K}\left(V_{\gamma}, \mathcal{H} \otimes L\right)
$$

for every $z \in Z$. In particular it is independent of $z$. Theorem 8.1 implies that there exists $k$ such that for each $u \in U$ the map

$$
T_{k, L_{u}}: J\left(L_{u}\right) \rightarrow I\left(\sigma_{k, L_{u}}\right)
$$

is injective. Note that the space of $K$-finite vectors in $I\left(\sigma_{k, L_{u}}\right)$ is the $K$-finite induced representation $\operatorname{Ind}_{M}^{K}\left(\sigma_{k, L_{\mid M}}\right)$ and hence independent of $u$. Let $\left(H_{1},\langle\ldots, \ldots\rangle\right)$ be the Hilbert space completion of $\operatorname{Ind}_{M}^{K}\left(\sigma_{k, L_{\mid M}}\right)$ corresponding to unitary induction from $M$ to $K$. This gives a smoothable analytic family of Hilbert representations of $G$ (Proposition 6.3),$\mu_{z}$. Proposition G.3 now implies the result.

\section{THE MAIN THEOREM}

Recall that $G$ is a real reductive group of inner type.

Theorem 9.1. Let $(\pi, V)$ be an analytic family of objects in $\mathcal{H}(\mathfrak{g}, K)$ based on the analytic manifold $X$. Let $x_{o} \in X$; then there exists $U$, an open neighborhood of $x_{o}$ in $X$, and a smoothable, continuous family of Hilbert representations $\left(\mu_{U}, H_{U}\right)$ such that the family $\left(d \mu_{U},\left(H_{U}\right)_{K}^{\infty}\right)$ is isomorphic with $\left(\pi_{\mid U}, V\right)$ (as a continuous family).

Proof. Let $U_{1}$ be an open neighborhood of $x_{o}$ in $X$ with compact closure. Then Theorem E.6 implies that there exists $F_{U_{1}}^{0} \subset \hat{K}$ a finite subset such that

$$
\pi_{x}\left(U\left(\mathfrak{g}_{\mathbb{C}}\right)\right) \sum_{\gamma \in F_{U}^{0}} V(\gamma)=V .
$$

Let $R^{0}=\sum_{\gamma \in F_{U}} V(\gamma) . R^{0}$ is invariant under the action $\pi_{x}(\mathbf{D})$ for all $x \in X$. This implies that $\left(\left.\left(\pi_{\mid U}\right)\right|_{\mathbf{D}}, R^{0}\right)$ defines an analytic family of objects in $W(\mathbf{D}, K)$ based on $U_{1}$. Let $J\left(R^{0}\right)$ be the corresponding $J$-family. Then we have the surjective analytic homomorphism of families

$$
J\left(R^{0}\right) \stackrel{T_{0}}{\rightarrow} V_{\mid U} \rightarrow 0
$$

with $T_{0}(x)$ mapping $J\left(R_{x}^{0}\right)$ onto $V$ for all $x \in U_{1}$. Let $\left(\sigma,\left(H^{0},(\ldots, \ldots)\right)\right)$ be the smoothable, continuous family of Hilbert representations based on $U_{1}$ corresponding to $J\left(R^{0}\right)$ as in Theorem 8.2. Let $U$ be an open neighborhood of $x_{o}$ contained in $U_{1}$ such that $\bar{U}$ is contractible. The theorem now follows from Proposition G.4

The main result is

Theorem 9.2. Let $T$ denote the inverse functor to the $K$-finite functor and let $(\pi, V)$ be a holomorphic family of objects in $\mathcal{H}(\mathfrak{g}, K)$ based on the connected complex manifold $X$. Then if $x, y \in X, T\left(\pi_{x}, V\right)=T\left(\pi_{y}, V\right)$ as subspaces of $\prod_{\gamma \in \hat{K}} V(\gamma)$ and if $T\left(\pi_{x} V\right)=\left(\lambda_{x}, W\right)$ then $(\lambda, W)$ is a holomorphic family of smooth Fréchet representations based on $X$.

Proof. The above theorem implies that there is an open covering, $\left\{U_{\alpha}\right\}$, of $X$ and for each $\alpha$ a continuous family of smoothable, admissible Hilbert representations based on $U_{\alpha},\left(\sigma_{\alpha}, H_{\alpha}\right)$, such that $\left(\left(d \sigma_{\alpha}\right)_{x},\left(H_{\alpha}\right)_{K}\right)=\left(\pi_{x}, V\right), x \in U_{\alpha}$. Proposition F.2 combined with Theorem F.5 implies that $\left(\sigma_{\alpha}, H_{\alpha}^{\infty}\right)$ (here $H_{\alpha}^{\infty}$ is 
the space of $C^{\infty}$ vectors with respect to $K$ ) is a holomorphic family of smooth Fréchet representations of $G$ based on $U_{\alpha}$. The isomorphism of categories immediately implies that if $x \in U_{\alpha} \cap U_{\beta}$ then $H_{\alpha}^{\infty}=H_{\beta}^{\infty}$ as subsets of $\prod_{\gamma \in \hat{K}} V(\gamma)$ and $\left.\left(\sigma_{\alpha}\right)_{x}(g)\right|_{H_{\alpha}^{\infty}}=\left.\left(\sigma_{\beta}(g)\right)_{x}\right|_{H_{\beta}^{\infty}}$ for all $g \in G$. Thus we can define $W=H_{\alpha}^{\infty}$ the common value for all $\alpha$ (since $X$ is connected) and if $x \in X$ then $\sigma_{x}(g)=\left(\sigma_{\alpha}\right)_{x}(g)$ for $\alpha$ such that $x \in U_{\alpha}$.

This (and its proof) can be interpreted in the following way:

Corollary 9.3. Let $T$ be the inverse functor to the $K$-finite functor $\mathcal{H} \mathcal{F}(G) \rightarrow$ $\mathcal{H}(\mathfrak{g}, K)$ and let $(\pi, V)$ be a holomorphic family of objects in $\mathcal{H}(\mathfrak{g}, K)$ over the connected complex manifold $X$. If $T\left(\left(\pi_{x}, V\right)\right)=\left(\lambda_{x}, \overline{V_{x}}\right)$ then

(1) For all $x, y \in X, \overline{V_{x}}=\overline{V_{y}}$ as subspaces of $\prod_{\gamma \in \hat{K}} V(\gamma)$ and as Fréchet spaces. Set $\bar{V}$ equal to the common value.

(2) The map $x, g, v \longmapsto \lambda_{x}(g) v$ is continuous from $X \times G \times \bar{V}$ to $\bar{V}$, linear in $v$ and $C^{\infty}$ in $g$ and holomorphic in $x$.

Using the results in Appendix $\mathrm{G}$ and the observation that if $V$ is an admissible smooth, Fréchet module of moderate growth then the topology of $V$ is given by norms corresponding to continuous inner products one has

Corollary 9.4. If $(\pi, V)$ and $(\sigma, W)$ are holomorphic families of admissible, smooth Fréchet modules over the complex manifold $X$ and if $T:(\pi, V) \rightarrow(\sigma, W)$ is a holomorphic family of homomorphisms of families (see Definition D.3) then image of $T$ (i.e.

$$
\left.x \mapsto\left(\sigma_{x \mid T_{x}(V)}, T_{x}(V)\right)\right)
$$

and the kernel of $T$ (i.e.

$$
\left.x \mapsto\left(\pi_{x \mid \operatorname{ker} T_{x}}, \operatorname{ker} T_{x}\right)\right)
$$

is a holomorphic family of admissible, smooth Fréchet modules over $X$.

\section{Appendix A. $G-C^{\infty}$ vectors And $K-C^{\infty}$ vectors}

Let $G$ be a real reductive group with a fixed maximal compact subgroup $K$ and let $\theta$ be the corresponding Cartan involution. Fix a symmetric bilinear form $B$ on $\operatorname{Lie}(G)$ such that $\langle X, Y\rangle=-B(\theta X, Y)$ is positive definite. Let $C$ and $C_{K}$ be the Casimir operators of $G$ and $K$ respectively corresponding to $B$ and we set $\Delta=C-2 C_{K}$. We observe that $\Delta=\sum X_{i}^{2}$ for $X_{1}, \ldots, X_{m}$ an orthonormal basis of $\operatorname{Lie}(G)$ relative to $\langle\ldots, \ldots\rangle$. As a left invariant operator on $G, \Delta$ is an elliptic and invariant under $K$. Let $(\pi, H)$ be a Hilbert representation of $G$ and set $V=\left(H^{\infty}\right)_{K}$. Let $Z$ be the completion of $V$ relative to the seminorms $q_{l}(v)=\left\|\Delta^{l} v\right\|, l=0,1,2$, $\ldots$ Then since $q_{0}=\|\ldots\|, Z$ can be looked upon as a subspace of $H$. Also $H^{\infty}$ is the completion of $V$ using the seminorms $s_{x}(v)=\|x v\|$ with $x \in U(\mathfrak{g})$. Thus $Z \supset H^{\infty}$.

Lemma A.1. $Z=H^{\infty}$. Furthermore, the topology on $H^{\infty}$ is given by the seminorms $q_{l}$.

Proof. We note that the second assertion is a direct consequence of the closed graph theorem (cf. [T] ) and the first assertion. We will now prove the first assertion. Let 
$v \in Z \subset H$. We must prove that $v \in H^{\infty}$. Let $v_{j} \in V$ be a sequence converging to $v$ in the topology of $Z$. Let $w \in H$; then for all $j$

$$
\Delta^{k}\left(\pi(g) v_{j}, w\right)=\left(\pi(g) \Delta^{k} v_{j}, w\right) .
$$

Set $p_{k}(v)=\sum_{j=0}^{k} q_{k}(v)$. Noting that $p_{l}\left(\Delta^{k} v\right)+p_{k-1}(v)=p_{l+k}(v)$ and $p_{l}(v) \leq$ $p_{l+1}(v)$, we see that for fixed $k$ the sequence $\left\{\Delta^{k} v_{j}\right\}_{j}$ converges to $u_{k}$ in $Z$.

We assert that the function $g \mapsto(\pi(g) v, w)$ is $C^{\infty}$. Since $w \in H$ is arbitrary this would imply that the map $g \mapsto \pi(g) v$ is weakly $C^{\infty}$. But a weakly $C^{\infty}$ map of a finite dimensional manifold into a Hilbert space is strongly $C^{\infty}$ (cf. [G2]). This is exactly the statement that $v$ is a $C^{\infty}$ vector. We now prove the assertion. We first show that if we look upon the continuous function $h(g)=(\pi(g) v, w)$ as a distribution on $G$ (using the Haar measure on $G$ ) then in the distribution sense

$$
\Delta^{k} h(g)=\left(\pi(g) u_{k}, w\right) .
$$

Indeed, let $f \in C_{c}^{\infty}(G)$; then

$$
\begin{gathered}
\int_{G} h(g) \Delta^{k} f(g) d g=\lim _{j \rightarrow \infty} \int_{G}\left(\pi(g) v_{j}, w\right) \Delta^{k} f(g) d g= \\
\lim _{j \rightarrow \infty} \int_{G} \Delta^{k}\left(\pi(g) v_{j}, w\right) f(g) d g=\lim _{j \rightarrow \infty} \int_{G}\left(\pi(g) \Delta^{k} v_{j}, w\right) f(g) d g= \\
\int_{G}\left(\pi(g) u_{k}, w\right) f(g) d g
\end{gathered}
$$

as asserted. Since $\Delta$ is elliptic, local Sobolev theory (cf. [T, Chapter 6]) implies that $h \in C^{\infty}(G)$.

Proposition A.2. If $(\pi, H)$ is an admissible Hilbert representation of $G$ such that there exists a polynomial

$$
f(x)=x^{m}-\sum_{j=0}^{m-1} c_{j} x^{j}
$$

with $f(C)=0$ on $H^{\infty}$ then the topology of $H^{\infty}$ is given by the semi-norms $p_{l}(v)=$ $\left\|\left(I+C_{K}\right)^{l} v\right\|, l=0,1,2, \ldots$ That is, the $K-C^{\infty}$ vectors, $H^{\infty_{K}}$ are the same as the $G-C^{\infty}$ vectors, $H^{\infty}$.

Proof. This result will be proved by induction on $m$. If $m=1$ then $C$ acts by $c=c_{0}$ on $H^{\infty}$. Note that $\Delta=C-2 C_{K}$. So if $v \in H^{\infty}$

$$
\begin{gathered}
\left\|\Delta^{k} v\right\|=\left\|\sum_{j=0}^{k}(-2)^{j}\left(\begin{array}{c}
k \\
j
\end{array}\right) C^{k-j} C_{K}^{j} v\right\| \leq \\
\sum_{j=0}^{k}(2)^{j}\left(\begin{array}{c}
k \\
j
\end{array}\right)|c|^{k-j}\left\|C_{K}^{j} v\right\| \leq \sum_{j=0}^{k}(2)^{j}\left(\begin{array}{c}
k \\
j
\end{array}\right)|c|^{k-j}\left\|\left(1+C_{K}\right)^{j} v\right\| .
\end{gathered}
$$

Now assume the result if the degree is $m-1 \geq 1$. Let $H^{\omega}$ denote the space of analytic vectors in $H^{\infty}$. The $K$-finite vectors $V=H_{K}$ are contained in $H^{\omega}$ since $H$ is admissible. If $c \in \mathbb{C}$ and $V_{c}=\{v \in V \mid C v=c v\} \neq 0$ then let $H_{1}$ be the Hilbert space completion of $V_{c}$; then $H_{1}$ is $G$-invariant, $\left(H_{1}\right)_{K}=V_{c}$ and $\left(H / H_{1}\right)_{K}=H_{K} / V_{c}$. Fix $c$ such that $V_{c} \neq 0$. The first part of the proof implies that the seminorms $p_{l}$ define the topology on $H_{1}^{\infty}$. The correspondence $U \rightarrow U^{\infty}$ is an exact functor from the category of strongly continuous Hilbert representations 
of $G$ to the category of smooth Fréchet modules [W, Proposition 4.4.1.11, p. 260]. Setting

$$
g(x)=\frac{f(x)}{x-c}
$$

we have $g(C)$ is zero on $\left(H / H_{1}\right)^{\infty}$ (since it is 0 on $V / V_{c}$ ). Thus we have the commutative diagram

$$
\begin{aligned}
& 0 \rightarrow H_{1}^{\infty} \rightarrow H^{\infty} \rightarrow\left(H / H_{1}\right)^{\infty} \rightarrow 0
\end{aligned}
$$

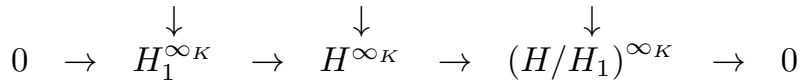

with the right-most and left-most vertical arrows isomorphisms. This implies that the middle vertical arrow is also an isomorphism completing the induction.

Corollary A.3. If $(\pi, H)$ is an admissible finitely generated Hilbert representation of $G$ then the $K-C^{\infty}$ vectors are the same as the $G-C^{\infty}$ vectors.

Proof. There exists a finite subset $F \subset \hat{K}$ such that $U(\mathfrak{g}) \sum_{\gamma \in F} H(\gamma)=H_{K}$. Clearly, $C \sum_{\gamma \in F} H(\gamma) \subset \sum_{\gamma \in F} H(\gamma)$. Let $p(x)$ be the minimal polynomial of $C_{\mid \sum_{\gamma \in F} H(\gamma)}$. Then $p(C)=0$ on $H_{K}$ and hence on $H^{\infty}$.

\section{Appendix B. Hilbert families}

Let $G$ be a locally compact topological group and let $X$ be a locally compact metric space. All Hilbert spaces in this appendix (indeed, in this paper) will be separable.

Definition B.1. A continuous family of Hilbert representations of $G$ over $X$ is a pair $(\sigma, H)$ with $H$ a Hilbert space and $\sigma: X \times G \rightarrow G L(H)$ (the continuous invertible operators on $H$ with the strong operator topology) a continuous map such that $\sigma_{x}(g)=\sigma(x, g)$ defines a representation of $G$ for every $x \in G$.

Lemma B.2 is Lemma 1.1.3 in RRG taking into account dependence on parameters. The proof is essentially the same using the local compactness of $X$.

Lemma B.2. Let $X$ be a locally compact metric space and let $H$ be a Hilbert space. Assume that for each $x \in X, \pi_{x}: G \rightarrow G L(H)$ (bounded invertible operators) such that

(1) If $\omega \subset X$ and $\Omega \subset G$ are compact subsets of $X$ and of $G$ respectively then there exists a constant $C_{\omega, \Omega}$ such that $\left\|\pi_{x}(g)\right\| \leq C_{\omega, \Omega}$ for $x \in \omega, g \in \Omega$.

(2) The map $x, g \rightarrow\left\langle\pi_{x}(g) v, w\right\rangle$ is continuous for all $v, w \in H$.

Then $(\pi, H)$ is a continuous family of representations of $G$ based on $X$ and conversely if $(\pi, H)$ is a continuous family of Hilbert representations then (1) and (2) are satisfied.

An immediate corollary is

Corollary B.3. Let $(\pi, H)$ be an admissible, continuous family of Hilbert representations of $G$ based on the locally compact metric space $X$. Set for each $x \in X$, $\hat{\pi}_{x}(g)=\pi_{x}\left(g^{-1}\right)^{*}$; then $(\hat{\pi}, H)$ is a continuous, admissible family of Hilbert representations of $G$ based on $X$. 


\section{Appendix C. Norms}

Let $\|g\|$ be a norm on $G$, that is, a continuous function from $G$ to $\mathbb{R}_{>0}$ (the positive real numbers) such that

(1) $\left\|k_{1} g k_{2}\right\|=\|g\|, k_{1}, k_{2} \in K, g \in G$,

(2) $\|x y\| \leq\|x\|\|y\|, x, y \in G$,

(3) The sets $\|g\| \leq r<\infty$ are compact.

(4) If $X \in \mathfrak{p}$ then if $t \geq 0$ then $\log \|\exp t X\|=t \log \|\exp X\|$.

If $(\sigma, V)$ is a finite dimensional representation of $G$ with compact kernel. Assume that $\langle\ldots, \ldots\rangle$ is an inner product on $V$ that is $K$-invariant and is such that the elements $\sigma(\exp (X))$ are self adjoint for $X \in \mathfrak{p}_{o}$. If $\|\sigma(g)\|$ is the operator norm of $\sigma(g)$ then $\|g\|=\|\sigma(g)\|$ is a norm on $G$. Taking the representation on $V \oplus V$ given by

$$
\left[\begin{array}{cc}
\sigma(g) & \\
& \sigma\left(g^{-1}\right)^{*}
\end{array}\right]
$$

then we may (and will) assume in addition

(5) $\|g\|=\left\|g^{-1}\right\|$.

Note that (5) implies that $\|g\| \geq 1$.

Using the same proof as Lemma 2.A.2.1 in RRG] (which we give for the sake of completeness) one can prove

Lemma C.1. If $(\pi, H)$ is a continuous family of Hilbert representations over $\omega$ a compact metric space then there exist constants $C_{\omega}, r_{\omega}$ such that

$$
\left\|\pi_{x}(g)\right\| \leq C_{\omega}\|g\|^{r_{\omega}} .
$$

Proof. Let

$$
B_{1}=\{g \in G \mid\|g\| \leq 1\} .
$$

If $v \in H$ and $(x, g) \in \omega \times B_{1}$ then $\sup \left\|\pi_{x}(g) v\right\|<\infty$ by strong continuity. The principle of uniform boundedness (cf. [RS, III.9, p. 81]) implies that there exists a constant, $R$, such that $\left\|\pi_{x}(g)\right\| \leq R$ for $(x, g) \in \omega \times B_{1}$. Let $r=\log R$. In particular if $k \in K$ then

$$
\left\|\pi_{x}(k g)\right\| \leq\left\|\pi_{x}(k)\right\|\left\|\pi_{x}(g)\right\| \leq R\left\|\pi_{x}(g)\right\| .
$$

Also,

Thus for all $k \in K, g \in G$

$$
\left\|\pi_{x}(g)\right\|=\left\|\pi_{x}\left(k^{-1}\right) \pi_{x}(k g)\right\| \leq R\left\|\pi_{x}(k g)\right\| .
$$

$$
R^{-1}\left\|\pi_{x}(g)\right\| \leq\left\|\pi_{x}(k g)\right\| \leq R\left\|\pi_{x}(g)\right\| .
$$

Let $X \in \mathfrak{p}, X \neq 0$ and let $j$ be such that

$$
j<\log \|\exp X\| \leq j+1,
$$

then

$$
\log \left\|\pi_{x}(\exp X)\right\| \leq \log \| \pi_{x}\left(\exp \left(\frac{X}{j+1}\right)\left\|^{j+1} \leq r(j+1) \leq r+r \log \right\| \exp X \| .\right.
$$

Thus

$$
\left\|\pi_{x}(\exp X)\right\| \leq R\|\exp X\|^{r}, X \in \mathfrak{p} .
$$

If $g \in G$ then $g=k \exp X$ with $k \in K$ and $X \in \mathfrak{p}$ so

$$
\left\|\pi_{x}(g)\right\|=\left\|\pi_{x}(k \exp X)\right\| \leq R^{2}\|\exp X\|^{r}=R^{2}\|g\|^{r} .
$$

Take $C_{\omega}=R^{2}$ and $r_{\omega}=r$. 


\section{Appendix D. Continuous and analytic families of $(\mathfrak{g}, K)$ modules}

Let $G$ be a reductive group with fixed maximal compact subgroup, $K$. In this section $X$ will denote a connected, paracompact real analytic or complex manifold.

Definition D.1. If $V$ is a vector space over $\mathbb{C}$ then a continuous, real analytic or holomorphic function from $X$ to $V$ is a map $f: X \rightarrow V$ such that for each $x \in X$ there exists $U$, an open neighborhood of $x$ in $X$ such that the following two conditions are satisfied:

(1) $\operatorname{dim} \operatorname{span}_{\mathbb{C}}\{f(x) \mid x \in U\}<\infty$.

(2) $f: U \rightarrow \operatorname{span}_{\mathbb{C}}\{f(x) \mid x \in U\}$ is respectively continuous, real analytic or holomorphic.

Definition D.2. A holomorphic, analytic or continuous family of admissible $(\mathfrak{g}, K)$ modules over $X$ is a pair, $(\mu, V)$, of an admissible $(\mathfrak{k}, K)$-module, $V$, and

$$
\mu: X \times U(\mathfrak{g}) \rightarrow \operatorname{End}(V)
$$

such that $x \mapsto \mu(x, y) v$ is respectively holomorphic, analytic or continuous for all $y \in U(\mathfrak{g}), v \in V$ and if we set $\mu_{x}(y)=\mu(x, y)$ for $y \in U(\mathfrak{g})$ then $\left(\mu_{x}, V\right)$ is an admissible $(\mathfrak{g}, K)$-module. It will be called a family of objects in $\mathcal{H}(\mathfrak{g}, K)$ over $X$ if each $\left(\mu_{x}, V\right)$ is finitely generated.

Definition D.3. If $(\lambda, V)$ and $(\mu, W)$ are analytic or continuous families of objects in $\mathcal{H}(\mathfrak{g}, K)$ over $X$ then a homomorphism of the family $(\lambda, V)$ to $(\mu, W)$ is a map

$$
T: X \rightarrow \operatorname{Hom}_{\mathbb{C}}(V, W)
$$

such that

(1) $x \mapsto T(x) v$ is an analytic or continuous map of $X$ to $W$ for all $v \in V$.

(2) $T(x) \in \operatorname{Hom}_{\mathcal{H}(\mathfrak{g}, K)}\left(V_{x}, W_{x}\right)$ with $V_{x}=\left(\lambda_{x}, V\right), W_{x}=\left(\mu_{x}, W\right)$.

Lemma D.4. Let $(\pi, H)$ be a continuous family of admissible Hilbert representations of $G$ over $X$ and denote by $d \pi_{x}$ the action of $\mathfrak{g}$ on $H_{K}^{\infty}$ (the $K$-finite $C^{\infty}$ vectors). Then $\left(d \pi, H_{K}\right)$ is a continuous family of admissible $(\mathfrak{g}, K)$-modules based on $X$.

Proof. If $\gamma \in \hat{K}$ then we will use the notation $C_{c}^{\infty}(\gamma ; G)$ for the space of all $f \in$ $C_{c}^{\infty}(G)$ such that

$$
d(\gamma) \int_{K} \chi_{\gamma}(k) f\left(k^{-1} g\right) d k=f(g), g \in G
$$

with $\chi_{\gamma}$ the character of $\gamma$. Then

$$
H(\gamma)=\pi_{x}\left(C_{c}^{\infty}(\gamma ; G)\right) H
$$

We also note that if $Y \in \mathfrak{g}, f \in C_{c}^{\infty}(\gamma ; G)$ and $v \in H$ then

$$
d \pi_{x}(Y) \pi_{x}(f) v=\pi_{x}(Y f) v
$$

with $Y f$ the usual action of $Y \in \mathfrak{g}$ on $C^{\infty}(G)$ as a left invariant vector field (that is differentiate on the right). Thus, if $v \in H_{K}$ and $y \in U\left(\mathfrak{g}_{\mathbb{C}}\right)$ then the map

$$
x \longmapsto d \pi_{x}(y) v
$$

is continuous. 


\section{Appendix E. Some results of Vincent van der Noort}

Throughout this section $Z$ will denote a connected real or complex analytic manifold. We will use the terminology analytic to mean complex analytic or real analytic depending on the context.

We continue the notation of the previous sections. In particular $G$ is a real reductive group of inner type.

We denote (as is usual) the standard filtration of $U(\mathfrak{g})$ by

$$
\cdots \subset U^{j}(\mathfrak{g}) \subset U^{j+1}(\mathfrak{g}) \subset \ldots
$$

Let $V$ be an admissible $(\operatorname{Lie}(K), K)$ module. We note that if $E \subset V$ is a finite dimensional $K$-invariant subspace of $V$ then there exists a finite subset $F_{j, E} \subset \hat{K}$ such that

$$
U^{j}(\mathfrak{g}) \otimes E \cong \sum_{\gamma \in F_{j, E}} m_{\gamma, j} V_{\gamma}
$$

If $v \in V$ we denote by $E_{v}$ the span of $K v$ in $V$.

The purpose of this section is to prove a theorem of van der Noort which first appeared in his thesis $[\mathrm{VdN}$. We include the details only because he is not expected to publish it. In his thesis he studied the holomorphic case. Our exposition follows his original line.

Fix a maximal torus, $T$, of $M$; then $\mathfrak{h}_{o}=\operatorname{Lie}(T) \oplus \mathfrak{a}$ is a Cartan subalgebra of $\mathfrak{g}_{o}$. As usual, set $\mathfrak{h}$ equal to the complexification of $\mathfrak{h}_{o}$. We parametrize the homomorphisms of $Z(\mathfrak{g})$ to $\mathbb{C}$ by $\chi_{\Lambda}$ for $\Lambda \in \mathfrak{h}^{*}$ using the Harish-Chandra parametrization. Since $M$ is compact we endow $\hat{M}$ with the discrete topology. Note that if $C$ is a compact subset (sorry of the over use of $C$, the Casimir operator will not appear in this section) of $\hat{M} \times \mathfrak{a}_{\mathbb{C}}^{*}$ then there exist a finite number of elements $\xi_{1}, \ldots, \xi_{r} \in \hat{M}$ and compact subsets, $D_{j}$, of $\mathfrak{a}_{\mathbb{C}}^{*}$ such that

$$
C=\cup_{j=1}^{r} \xi_{j} \times D_{j} .
$$

If $\xi \in \hat{M}$ and $\nu \in \mathfrak{a}_{\mathbb{C}}^{*}$ then set $\sigma_{\xi, \nu}(\operatorname{man})=\xi(m) a^{\nu+\rho}\left(\rho(H)=\frac{1}{2} \operatorname{tr}\left(\operatorname{ad} H_{\mid \operatorname{Lie}(N)}\right)\right)$, $H \in \mathfrak{a}), a^{\nu}=\exp (\nu(H)) a=\exp (H), \xi$ is taken to be a representative of the class $\xi . H^{\xi, \nu}$ is $I\left(\sigma_{\xi . \nu}\right)$ which equals as a $K$-module $H^{\xi}=\operatorname{Ind}_{M}^{K}(\xi)$. If $f \in H^{\xi}$ set $f_{\nu}(n a k)=a^{\nu+\rho} f(k), n \in N, a \in A, k \in K . A_{\bar{P}}(\nu)$ is the corresponding Kunze-Stein intertwining operator (cf. W1, 8.10.18. p. 241]).

Proposition E.1. Let $\xi \in \widehat{M}$ and let $\Omega \subset \mathfrak{a}_{\mathbb{C}}^{*}$ be compact. There exists a finite set $F \subset \widehat{K}$ such that $\pi_{\xi, \nu}(U(\mathfrak{g}))\left(\sum_{\gamma \in F} H^{\xi}(\gamma)\right)=H^{\xi}$ for all $\nu \in \Omega$.

The proof of this result will use the following

Lemma E.2. If $\nu_{o} \in \mathfrak{a}_{\mathbb{C}}^{*}, \xi \in \hat{M}$ then there exists an open neighborhood of $\nu_{o}, U_{\nu_{o}}$, and a finite subset $F=F_{\nu_{o}}$ of $\widehat{K}$ such that $\pi_{\xi, \nu}(U(\mathfrak{g}))\left(\sum_{\gamma \in F} H^{\xi}(\gamma)\right)=H^{\xi}$ for all $\nu \in U_{\nu_{o}}$.

Proof. If $\gamma \in \hat{K}$ fix $W_{\gamma} \in \gamma$. If $\operatorname{Re}(\nu, \alpha)>0$ for all $\alpha \in \Phi^{+}$and if $\gamma \in \widehat{K}$ and $A_{\bar{P}}(\nu) H^{\xi}(\gamma) \neq 0$ then $\pi_{\xi, \nu}(U(\mathfrak{g}))\left(H^{\xi}(\gamma)\right)=H^{\xi}$ (cf. [RRG, Theorem 5.4.1 (1)]). Fix such a $\gamma_{\nu}$ (which always exists since the operator $A_{\bar{P}}(\nu) \neq 0$ ); take $F_{\nu}=\left\{\gamma_{\nu}\right\}$ and $U_{\nu}$ an open neighborhood of $\nu$ such that $A_{\bar{P}}(\mu) H^{\xi}\left(\gamma_{\nu}\right) \neq 0$ for $\mu \in U$. Let $\nu \in \mathfrak{a}_{\mathbb{C}}^{*}$ be arbitrary. There exists a positive integer, $k$, such that $\operatorname{Re}(\nu+k \rho, \alpha)>0$ for all $\alpha \in \Phi^{+}$and such that $k \rho$ is the highest weight of a finite dimensional spherical 
representation, $V^{k \rho}$, of $G$ relative to $\mathfrak{a}$. The lowest weight of $V^{k \rho}$ relative to $\mathfrak{a}$ is $-k \rho$ and $M$ acts trivially on that weight space; thus $H_{K}^{\xi, \nu+k \rho} \otimes V^{k \rho}$ has $H_{K}^{\xi, \nu}$ as a quotient representation (see [W1, 8.5.14,15]). Take $F_{\nu}$ to be the set of $K$-types that occur in both $W_{\gamma_{\nu+k \rho}} \otimes V^{k \rho}$ and $H^{\xi}$ and $U_{\nu}=U_{\nu+k \rho}-k \rho$.

We now prove the proposition. By the lemma above for each $\nu \in \Omega$ there exist $F_{\nu}$ and $U_{\nu}$ as in the statement of the lemma. The $U_{\nu}$ form an open covering of $\Omega$ which is assumed to be compact. Thus there exist a finite number $\nu_{1}, \ldots, \nu_{r} \in \Omega$ such that

$$
\Omega \subset \cup_{i=1}^{r} U_{\nu_{i}} .
$$

Take $F=\cup_{i-1}^{r} F_{\nu_{i}}$. This proves the proposition.

Lemma E.3. Let $\chi_{\xi, \nu}$ denote the infinitesimal character of $\pi_{\xi, \nu}$. If $C$ is a compact subset of $\mathfrak{h}^{*}$ then

$$
\left\{(\xi, \nu) \in\left\{\hat{M} \times \mathfrak{a}_{\mathbb{C}}^{*} \mid \chi_{\xi}, \nu=\chi_{\Lambda}, \Lambda \in C\right\}\right.
$$

is compact.

Proof. Fix a system of positive roots for $\left(M^{0}, T\right)\left(M^{0}\right.$ the identity component of $M)$. If $\lambda_{\xi}$ is the highest weight of $\xi$ relative to this system of positive roots and if $\rho_{M}$ is the half sum of these positive roots then $\chi_{\xi, \nu}=\chi_{\Lambda}$ with $\Lambda=\lambda_{\xi}+\rho_{M}+\nu$. This implies the lemma.

The following result is the reason for the assumption of analyticity.

Lemma E.4. Let $(\pi, V)$ be an analytic family of admissible $(\mathfrak{g}, K)$ modules over $Z$. Assume that there exists $z_{0} \in Z$ such that $\left(\pi_{z_{o}}, V\right)$ is finitely generated. If $T \in Z(\mathfrak{g})$ then there exist analytic functions $a_{0, T}, \ldots, a_{n-1, T}$ on $Z$ such that if

$$
f_{T}(z, x)=x^{n}+\sum_{j=0}^{n-1} a_{j, T}(z) x^{j}
$$

for $z \in Z$ then $f_{T}\left(z, \pi_{z}(T)\right)=0$ for all $z \in Z$.

Proof. Let $F$ be a finite subset of $\hat{K}$ such that $\pi_{z_{0}}(U(\mathfrak{g})) \sum_{\gamma \in F} V(\gamma)=V$. Let $L=$ $\sum_{\gamma \in F} V(\gamma)$. Then we define the functions $a_{j}$ the by the formula

$$
f(z, x)=\operatorname{det}\left(x I-\pi_{z}(T)_{\mid L}\right)=x^{n}+\sum_{j=0}^{n-1} a_{j}(z) x^{j} .
$$

The Cayley-Hamilton theorem implies that $h(z)=T^{n}+\sum_{j=0}^{n-1} a_{j}(z) T^{j} \in Z(\mathfrak{g})$ vanishes on $L$. Let $\gamma \in \hat{K}$; then there exist $x_{1}, \ldots, x_{r} \in U(\mathfrak{g})$ and $v_{1}, \ldots, v_{r} \in L$ such that $\left\{\pi_{z_{0}}\left(x_{i}\right) v_{i}\right\}_{i=1}^{r}$ is a basis of $V(\gamma)$. Let $P_{\gamma}$ be the projection onto the $\gamma$-isotypic component of $V$. Thus

$$
\left(P_{\gamma} \pi_{z}\left(x_{1}\right) v_{1}\right) \wedge\left(P_{\gamma} \pi_{z}\left(x_{2}\right) v_{2}\right) \wedge \cdots \wedge\left(P_{\gamma} \pi_{z}\left(x_{r}\right) v_{r}\right) \in \wedge^{r} V(\gamma)
$$

(a one dimensional space) is non-zero for $z=z_{0}$. This implies that there exists an open neighborhood, $U$, of $z_{0}$ in $\Omega$ such that

$$
P_{\gamma} \pi_{z}\left(x_{1}\right) v_{1}, P_{\gamma} \pi_{z}\left(x_{2}\right) v_{2}, \ldots, P_{\gamma} \pi_{z}\left(x_{r}\right) v_{r}
$$

is a basis of $V(\gamma)$ for $z \in U$. Since

$$
h(z) P_{\gamma} \pi_{z}\left(x_{i}\right) v_{i}=P_{\gamma} \pi_{z}\left(x_{i}\right) h(z) v_{i}=0,
$$


we have $h(z) V(\gamma)=0$ for $z \in U$. The connectedness of $Z$ and the analyticity imply that $h(z) V(\gamma)=0$ for $z \in Z$. Thus since $\gamma$ is arbitrary $h(z)=0$ for all $z \in Z$. This proves the Lemma.

If $V$ is a $(\mathfrak{g}, K)$-module then set $\operatorname{ch}(V)$ equal to the set of $\Lambda \in \mathfrak{h}^{*}$ such that there exists $v \in V$ with $T v=\chi_{\Lambda}(T) v$ for all $T \in Z(\mathfrak{g})$.

Corollary E.5. Keep the notation and assumptions of the previous lemma, If $\omega \subset$ $Z$ is compact then there exists a compact subset $C_{\omega}$ of $\mathfrak{h}^{*}$ such that $\operatorname{ch}\left(\pi_{z}, V\right) \subset C_{\omega}$ for all $z \in \omega$.

Proof. Let $T_{1}, \ldots, T_{m}$ be a generating set for $Z(\mathfrak{g})$ and let $f_{j}(z, x)=f_{T_{j}}(z, x)$ be the function in the previous lemma corresponding to $T_{j}$. Then

$$
f_{j}(z, x)=x^{n_{j}}+\sum_{i=0}^{n_{j}-1} a_{j, i}(z) x^{j}
$$

with $a_{j, i}$ analytic in $Z$. If $\chi_{\Lambda} \in \operatorname{ch}\left(\pi_{z}, V\right)$ then

$$
\left|\chi_{\Lambda}\left(T_{j}\right)\right| \leq \max _{0 \leq i<n_{j}}\left|a_{j, i}(z)\right|+1
$$

(cf. [RRG, 7.A.1.3]). If $L \subset Z$ is compact then there exists a constant $r<\infty$ such that $\left|a_{j, i}(z)\right| \leq r$ for all $i, j$ and $z \in L$. This implies the corollary.

Theorem E.6. Let $(\pi, V)$ be an analytic family of admissible $(\mathfrak{g}, K)$ modules over $Z$. Assume that there exists $z_{0} \in Z$ such that $\left(\pi_{z_{0}}, V\right)$ is finitely generated. If $\omega$ is a compact subset of $Z$ then there exists $S_{\omega} \subset \hat{K}$ a finite subset such that if $y \in \omega$ then

$$
\pi_{y}(U(\mathfrak{g}))\left(\sum_{\gamma \in S_{\omega}} V(\gamma)\right)=V .
$$

Proof. Let $C_{\omega}$ as in the above corollary for $\omega$. Let

$$
X=\left\{(\xi, \nu) \in \hat{M} \times \mathfrak{a}_{\mathbb{C}}^{*} \mid \chi_{\xi, \nu}=\chi_{\Lambda}, \Lambda \in C_{\omega}\right\} .
$$

$X$ is compact so there exist $\xi_{1}, \ldots, \xi_{r} \in \hat{M}$ and $D_{1}, \ldots, D_{r}$, compact subsets of $\mathfrak{a}_{\mathbb{C}}^{*}$, such that $X=\cup_{j} \xi_{j} \times D_{j}$. Let $S_{j} \subset \hat{K}$ be the finite set corresponding to $\xi_{j} \times D_{j}$ in Proposition E.1 Set $S_{\omega}=\cup S_{j}$. Let $L_{1} \subset L_{2} \subset \cdots \subset L_{j} \subset \ldots$ be an exhaustion of the $K$-types of $V$ with each $L_{j}$ finite.

We will use the notation $V_{y}$ for the $(\mathfrak{g}, K)$-module $\left(\pi_{y}, V\right)$. Let $y \in C$. Set $W_{j}=\pi_{y}(U(\mathfrak{g}))\left(\sum_{\gamma \in L_{j}} V(\gamma)\right)$; then $W_{j} \subset W_{j+1}$ and $\cup W_{j}=V$. Each $W_{j}$ is finitely generated and admissible, hence of finite length. Therefore $V_{y}$ has a finite composition series

or a countably infinite composition series

$$
0=V_{y}^{0} \subset V_{y}^{1} \subset \cdots \subset V_{y}^{N}
$$

$$
0=V_{y}^{0} \subset V_{y}^{1} \subset \cdots \subset V_{y}^{n} \subset V_{y}^{n+1} \subset \cdots
$$

with $V_{y}^{i} / V_{y}^{i-1}$ irreducible. Thus by the dual form of the subrepresentation theorem there exists for each $i, \xi_{i} \in \hat{M}$ and $\nu_{i} \in \mathfrak{a}_{\mathbb{C}}^{*}$ so that $V_{y}^{i} / V_{y}^{i-1}$ is a quotient of $\left(\pi_{\xi_{i}, \nu_{i}}, H^{\xi_{i}, \nu_{i}}\right)$. Observe that $\left(\xi_{i}, \nu_{i}\right) \in X$. Thus $V_{y}^{i} / V_{y}^{i-1}\left(\gamma_{i}\right) \neq 0$ for some $\gamma_{i} \in S_{\omega}$. Let $L$ be a quotient module of $V_{y}$. Then $L=V_{y} / U$ with $U$ a submodule of $V_{y}$. There must be an $i$ such that $V_{y}^{i} /\left(V_{y}^{i-1} \cap U\right) \neq 0$. Let $i$ be minimal subject to this 
condition. Then $V_{y}^{i-1} \subset U$. Thus $V_{y}^{i} / V_{y}^{i-1}$ is a submodule of $L$. Hence $L(\gamma) \neq 0$ for some $\gamma \in S_{\omega}$. This implies that

$$
\pi_{y}(U(\mathfrak{g}))\left(\sum_{\lambda \in S_{\omega}} V(\lambda)\right)=V .
$$

Indeed, we have shown that

$$
\left(V_{y} / \pi_{y}(U(\mathfrak{g}))\left(\sum_{\lambda \in S_{\omega}} V(\lambda)\right)\right)(\gamma)=0, \gamma \in S_{\omega}
$$

Corollary E.7. (To the proof) Let $(\pi, V)$ be an analytic family of finitely generated admissible $(\mathfrak{g}, K)$ modules over $Z$. Let $W \subset Z$ be compact . Let for each $z \in W$, $U_{z}$ be a $(\mathfrak{g}, K)$-submodule of $V_{z}$. Then there exists a finite subset $F_{W} \subset \hat{K}$ such that

$$
\pi_{z}(U(\mathfrak{g}))\left(\sum_{\gamma \in F_{W}} U_{z}(\gamma)\right)=U_{z}
$$

Proof. In the proof of the theorem above all that was used was that the set of possible infinitesimal characters is compact.

\section{Appendix F. Continuous And holomorphic FAmilies OF SMOOTH FRÉCHET REPRESENTATIONS}

Let $G$ be a reductive group with fixed maximal compact subgroup, $K$. Let $\mathcal{F}(G)$ denote the category of smooth Fréchet representations (here, as usual, smooth means that the map $g \mapsto \pi(g) v$ is $\left.C^{\infty}\right)$.

Definition F.1. A continuous family of objects in $\mathcal{F}(G)$ over a metric space $X$ is a pair $(\pi, V)$ of a Fréchet space $V$ and a continuous map

$$
\pi: X \times G \rightarrow \operatorname{End}(V)
$$

(here $\operatorname{End}(V)$ is the algebra of continuous operators on $V$ with the strong topology) such that for each $x \in X$, if $\pi_{x}(g)=\pi(x, g)$ then $\left(\pi_{x}, V\right) \in \mathcal{F}(G)$. We will say that the family has local uniform moderate growth if for each $\omega$ a compact subset of $X$ and each continuous seminorm on $V, p$, there exists a continuous seminorm $q_{\omega}$ on $V$ and $r_{\omega}$ such that if $v \in V$ then

$$
p\left(\pi_{x}(g) v\right) \leq q_{\omega}(v)\|g\|^{r_{\omega}} .
$$

Proposition F.2. If $(\pi, H)$ is a continuous family of Hilbert representations over the analytic manifold $X$ such that the representations $\pi_{x \mid K}$ are the same for all $x \in X$ (we denote this common value by $\pi(k)$ ) and the representations $\left(d \pi_{x}, H_{K}^{\infty}\right)$ form an analytic family of objects in $\mathcal{H}(\mathfrak{g}, K)$, then

(1) The space of $C^{\infty}$ vectors in $H$ with respect to $\pi_{x}$ is equal to the space of $C^{\infty}$ vectors of the representation $(\pi, H)$. of $K$.

(2) Assume that for each, $\omega \subset X$, compact, and $u \in U(\mathfrak{g})$ there exist constants $C_{\omega, v}, n_{\omega, u}$ such that

$$
\left\|d \pi_{y}(u) v\right\| \leq C_{\omega, u}\left\|d \pi\left(1+C_{K}\right)^{n_{\omega, u}} v\right\|
$$


for $v \in H^{\infty}$. Then $x \mapsto\left(\pi_{x}, H^{\infty}\right)$ is a continuous family of smooth Fréchet representations of local uniform moderate growth.

Proof. (1) follows from Lemma E.4 and Proposition A.2

We now prove (2) To prove the continuity assertion we need to show that if $l>0$ and $x_{o} \in X$ then

$$
\lim _{x \rightarrow x_{o}}\left\|d \pi\left(1+C_{K}\right)^{l}\left(\pi_{x}(g)-\pi_{x_{o}}(g)\right) v\right\|=0 .
$$

Let $\lambda_{\gamma}$ be the eigenvalue of $C_{K}$ on $V(\gamma)$. Recall that if $v \in H^{\infty}=\sum_{\gamma} v_{\gamma}$ with $v_{\gamma} \in H(\gamma)$ and for each $r$ there exists a constant $C_{r, v}$ such that

$$
\left\|v_{\gamma}\right\| \leq C_{v, r}\left(1+\lambda_{\gamma}\right)^{-r}
$$

As is well known

$$
\sum_{\gamma \in \hat{K}}\left(1+\lambda_{\gamma}\right)^{-r}<\infty
$$

if $r>\frac{\operatorname{dim} T}{2}$ with $T$ is a maximal torus of $K$. Fix $l>0$ and $x_{o}$ in $X$. Let $F \subset \widehat{K}$; if $u \in H^{\infty}$ set $u(F)=\sum_{\gamma \in F} u_{\gamma}$. If $F^{c}=\widehat{K}-F$, then $u=u(F)+u\left(F^{c}\right)$. If $u \in H^{\infty}$ then

$$
d \pi\left(1+C_{K}\right)^{l} \pi_{x}(g) u=\pi_{x}(g) d \pi_{x}\left(A d(g)^{-1}\left(1+C_{K}\right)^{l}\right) u .
$$

Let $z_{1}, \ldots, z_{d_{l}}$ be a basis of $U^{2 l}(\mathfrak{g})$. Then

$$
A d(g)^{-1}\left(1+C_{K}\right)^{l}=\sum a_{i}(g) z_{i}
$$

with $a_{i}$ real analytic on $G$. Thus

$$
d \pi\left(1+C_{K}\right)^{l} \pi_{x}(g) v=\pi_{x}(g) \sum a_{i}(g) d \pi_{x}\left(z_{i}\right) u .
$$

Note that there exists $C_{1}, m$ such that $\left|a_{i}(g)\right| \leq C_{1}\|g\|^{m}$ for all $i$. Now fix $x_{o} \in X$ and fix $U$ a neighborhood of $x_{o}$ with compact closure. Then (Lemma C.1)

$$
\left\|\pi_{x}(g) u\right\| \leq C_{2}\|g\|^{m_{1}}\|u\|, x \in U, u \in H .
$$

Let $v \in H^{\infty}$. Let for $N>0, F_{N}=\left\{\gamma \in \widehat{K} \mid \lambda_{\gamma} \leq N\right\}$; then $F_{N}$ is a finite set. Let $r=\frac{\operatorname{dim} T}{2}+1$. Set $n=\max _{i} n_{u_{i}, \omega}$ with $\omega$ the closure of $U$ and $C_{3}=\max C_{u_{i}, \omega}$. If $x \in U$

$$
\begin{aligned}
& \left\|d \pi\left(1+C_{K}\right)^{l}\left(\pi_{x}(g)-\pi_{x_{o}}(g)\right) v\left(F_{N}^{c}\right)\right\|^{2} \\
& \quad \leq 2 d_{l}^{2} C_{2}^{2} C_{1}^{2} C_{3}^{2}\|g\|^{m+m_{1}} \sum_{\gamma \notin F_{N}}\left(1+\lambda_{\gamma}\right)^{2 l+2 n}\left\|v_{\gamma}\right\|^{2} .
\end{aligned}
$$

Also

so

$$
\left\|v_{\gamma}\right\| \leq C_{v, m}\left(1+\lambda_{\gamma}\right)^{-m}
$$

$$
\sum_{\gamma \notin F_{N}}\left(1+\lambda_{\gamma}\right)^{2 l+2 n}\left\|v_{\gamma}\right\|^{2} \leq C_{v, m} \sum_{\gamma \notin F_{N}}\left(1+\lambda_{\gamma}\right)^{2 l-m} .
$$

Choose $m=2 l+r+2 n+s$ with $s \geq 1$. Then

$$
\sum_{\gamma \notin F_{N}}\left(1+\lambda_{\gamma}\right)^{2 l+2 n}\left\|v_{\gamma}\right\|^{2} \leq N^{-s} C_{v, m} \sum_{\gamma \notin F_{N}}\left(1+\lambda_{\gamma}\right)^{-r} \leq N^{-s} C_{v, m} \sum_{\gamma \notin \widehat{K}}\left(1+\lambda_{\gamma}\right)^{-r} .
$$

We therefore have $\left(C_{4}=C_{v, m} \sum_{\gamma \notin \widehat{K}}\left(1+\lambda_{\gamma}\right)^{-r}\right)$

$$
\left\|d \pi\left(1+C_{K}\right)^{l}\left(\pi_{x}(g)-\pi_{x_{o}}(g)\right) v\left(F_{N}^{c}\right)\right\|^{2} \leq 2 d_{l}^{2} C_{2}^{2} C_{1}^{2} C_{3}^{2}\|g\|^{m+m_{1}} C_{4} N^{-s} .
$$


Let $\varepsilon>0$ be and let $\omega_{1}$ be a compact subset of $G$. Choose $N$ so that

$$
2 d_{l}^{2} C_{2}^{2} C_{1}^{2} C_{3}^{2}\|g\|^{m+m_{1}} C_{4} N^{-s}<\frac{\varepsilon^{2}}{4}
$$

for $g \in \omega_{1}$. Now if $x \in U$ then

$$
\begin{aligned}
& \left\|d \pi\left(1+C_{K}\right)^{l}\left(\pi_{x}(g)-\pi_{x_{o}}(g)\right) v\right\| \\
& \quad \leq\left\|d \pi\left(1+C_{K}\right)^{l}\left(\pi_{x}(g)-\pi_{x_{o}}(g)\right) v\left(F_{N}\right)\right\|+\left\|d \pi\left(1+C_{K}\right)^{l}\left(\pi_{x}(g)-\pi_{x_{o}}(g)\right) v\left(F_{N}^{c}\right)\right\| \\
& \quad<\left\|d \pi\left(1+C_{K}\right)^{l}\left(\pi_{x}(g)-\pi_{x_{o}}(g)\right) v\left(F_{N}\right)\right\|+\frac{\varepsilon}{2} .
\end{aligned}
$$

The function of $x,\left\|d \pi\left(1+C_{K}\right)^{l}\left(\pi_{x}(g)-\pi_{x_{o}}(g)\right) v\left(F_{N}\right)\right\|^{2}$ is by our assumption real analytic in $x, g$ and equal to 0 at $x=x_{o}$. Hence there exists a neighborhood, $W$, of $x_{o}$ in $U$ such that if $g \in \omega_{1}$ and $x \in W$ then

$$
\left\|d \pi\left(1+C_{K}\right)^{l}\left(\pi_{x}(g)-\pi_{x_{o}}(g)\right) v\left(F_{N}\right)\right\|^{2}<\frac{\varepsilon^{2}}{4} .
$$

This completes the proof of continuity. We leave the condition of uniform moderated growth to the reader (what is needed is in the above argument).

Definition F.3. A continuous family of Hilbert representations of $G,(\pi, H)$, over $X$ will be called smoothable if for each compact subset $\omega \subset X, u \in U(\mathfrak{g})$ there exists $C_{\omega, u}, n_{\omega, u}$ such that

$$
\left\|d \pi_{y}(u) v\right\| \leq C_{\omega, u}\left\|d \pi\left(1+C_{K}\right)^{n_{\omega, u}} v\right\|
$$

for $y \in \omega, v \in H^{\infty}$.

Definition F.4. A holomorphic family of objects in $\mathcal{F}(G)$ over the complex manifold $X$ is a continuous family $(\pi, V)$ such that the map $x \longmapsto \pi_{x}(g) v$ is holomorphic from $X$ to $V$ for all $g \in G, v \in V$.

Theorem F.5. If $(\pi, V)$ is a continuous family of smooth Fréchet representations over the complex manifold $X$ such that $\left(d \pi, V_{K}\right)$ is a holomorphic family of objects in $\mathcal{H}(\mathfrak{g}, K)$ then $(\pi, V)$ is a holomorphic family of objects in $\mathcal{F}(G)$ over $X$.

We will use Lemma F.6 in the proof.

Lemma F.6. Let $X$ be a complex $n$-manifold, $G$ be connected and let

$$
f: X \times G \rightarrow \mathbb{C}
$$

be continuous and real analytic in $G$. If $z f(x, e)$ is holomorphic in $x \in X$ for all $z \in U(\mathfrak{g})$ (here $z$ is acting as left invariant differential operators on the $G$ the second factor) then $f$ is holomorphic in $X$.

Proof. Let $x \in X$ and let $z_{1}, \ldots, z_{n}$ be local coordinates on an open neighborhood, $U$, of $x$ in $X$ such that if $\psi=\left(z_{1}, \ldots, z_{n}\right)$ then $\psi(x)=0$ and $\psi(U) \supset \bar{D}^{n}$ with $D$ (resp. $\bar{D})$ the (resp. closed) unit disk in $\mathbb{C}$. For simplicity we may assume that $X=\psi(U)$. Define for $z \in D$

$$
h(z, g)=\frac{1}{(2 \pi i)^{n}} \int_{\left(S^{1}\right)^{n}} \frac{f(u, g)}{\prod\left(u_{i}-z_{i}\right)} d u_{1} \cdots d u_{n} .
$$

Then $h(z, g)$ is holomorphic in $z$ on $D$. By our assumption $u h(z, e)=u f(z, e)$ for $u \in U(\mathfrak{g}), z \in D$. Since $f$ is analytic in $G$ and $G$ is connected $h=f$ on $D$. 
We will now prove the theorem. Let $\lambda \in V^{\prime}$. If $v \in V_{K}$ then the function

$$
f(x, g)=\lambda\left(\pi_{x}(g) v\right)
$$

on $X \times G$ is continuous and real analytic in $G$. Now

$$
u f(x, e)=\lambda\left(d \pi_{x}(u) v\right)
$$

which is holomorphic in $x$. Thus if $v \in V_{K}$ then

$$
x \mapsto \lambda\left(\pi_{x}(g) v\right)
$$

is holomorphic in $x$. Let $x \in X$ and $U$, $\psi$, etc. be as in the previous lemma. Set

$$
h(z, v)=\frac{1}{(2 \pi i)^{n}} \int_{\left(S^{1}\right)^{n}} \frac{\lambda\left(\pi_{u}(g) v\right)}{\prod\left(u_{i}-z_{i}\right)} d u_{1} \cdots d u_{n}
$$

for $v \in V, z \in D^{n}$. Then $h(z, v)$ is holomorphic in $z$ and continuous in $v$. Furthermore, the first part of this proof showed that $h(z, v)=\lambda\left(\pi_{z}(g) v\right)$ if $v \in V_{K}$. Since $V_{K}$ is dense in $V$ this implies that

$$
z \mapsto \lambda\left(\pi_{z}(g) v\right)
$$

is holomorphic in $z$. Grothendieck G1 has shown that a weakly holomorphic map of a complex manifold to a Fréchet space is strongly holomorphic thus completing the proof.

\section{Appendix G. Functorial properties of Hilbert families}

In this section we will analyze Hilbert globalizations of subfamilies and quotient families of Harish-Chandra modules.

Lemma G.1. Let $(\tau, V)$ be a finite dimensional continuous representation of $K$ and let $X$ be a metric space. If $u \in X$ let $\langle\ldots, \ldots\rangle_{u}$ be an inner product on $V$ such that $\tau(k)$ acts unitarily with respect to $\langle\ldots, \ldots\rangle_{u}$ for $k \in K$ and such that the map $u \longmapsto\langle v, w\rangle_{u}$ is continuous (resp. real analytic) for all $v, w \in V$. Then there exists, for each $u$ an ordered orthonormal basis of $V, e_{1}(u), \ldots, e_{n}(u)$ such that the map $u \longmapsto e_{i}(u)$ is continuous (resp. real analytic) and the matrix of $\tau(k)$ with respect to $e_{1}(u), \ldots, e_{n}(u)$ is independent of $u$. Furthermore, if $X$ is a compact, contractible metric space and $(\sigma . W)$ is a finite dimensional continuous representation of $K$ and $u \longmapsto B(u) \in \operatorname{Hom}_{K}(V, W)$ is continuous and surjective for $u \in X$ then $e_{1}(u), \ldots, e_{r}(u)$ with $r=\operatorname{dim} V-\operatorname{dim} W$ can be taken in $\operatorname{ker} B(u)$.

Proof. Fix an inner product, $(\ldots, \ldots)$, on $V$ such that $\tau$ is unitary. Then there exists a positive definite Hermitian operator (with respect to $(\ldots, \ldots)), A(u)$, such that $\langle v, w\rangle_{u}=(A(u) v, w), v, w \in V$ and $A(u)$ is continuous (resp. real analytic) in $u$ and satisfying

$$
\tau(k)^{-1} A(u) \tau(k)=A(u), u \in X, k \in K .
$$

Set $S(u)=A(u)^{\frac{1}{2}}$; then $\langle v, w\rangle_{u}=(S(u) v, S(u) w)$. Thus if $T(u)=S(u)^{-\frac{1}{2}}$ then $\tau(k) T(u)=T(u) \tau(k), k \in K, u \longmapsto T(u)$ is continuous (resp. real analytic) and

$$
\langle T(u) v, T(u) w\rangle_{u}=(v, w), v, w \in V .
$$

Let $e_{1}, \ldots, e_{n}$ be an (ordered) orthonormal basis of $V$ with respect to $(\ldots, \ldots)$; then $e_{1}(u)=T(u) e_{1}, \ldots, e_{n}(u)=T(u) e_{n}$ is an orthonormal basis of $V$ with respect to $\langle\ldots, \ldots\rangle_{u}$. If $\tau(k) e_{i}=\sum k_{j i} e_{j}$ then

$$
\tau(k) e_{i}(u)=\tau(k) T(u) e_{i}=T(u) \tau(k) e_{i}=\sum k_{j i} T(u) e_{j} .
$$


To prove the second assertion note that $u \rightarrow \operatorname{ker} B(u)$ is a $K$-vector bundle over $X$ (see Lemma G.2). Since $X$ is compact and contractible the bundle is a trivial $K$-vector bundle [A, Lemma 1.6.4]. Thus there is a representation $(\mu, Z)$ of $K$ and a continuous map $u \longmapsto L(u) \in \operatorname{Hom}_{K}(Z, V)$ such that $L(u) Z=\operatorname{ker} B(u)$ and $L(u)$ is injective. Notice that $B(u):(\operatorname{ker} B(u))^{\perp} \rightarrow W$ is a $K$-module isomorphism. Now pull back the inner product $\langle\ldots, \ldots\rangle_{u}$ to $Z$ using $L(u)$ getting a $K$-invariant inner product, $(\ldots,,,,)_{u}$, on $Z$ and push the restriction of the inner product to $W$ using $B(u)$ getting a $K$-invariant inner product $(\ldots, \ldots)_{u}^{1}$ on $W$. Now apply the first part of the lemma to get an orthonormal basis $f_{1}(u), \ldots, f_{r}(u)$ of $Z$ with respect to $(\ldots, \ldots)_{u}$ and an orthonormal basis $f_{r+1}(u), \ldots, f_{n}(u)(n=\operatorname{dim} V)$ with respect to $(\ldots, \ldots)_{u}^{1}$ such that the matrices of the action of $K$ with respect to each of these bases is constant. Take $e_{i}(u)=L(u) f_{i}(u)$ for $i=1, \ldots, r$ and $e_{i}(u)=\left(B(u)_{\left.\right|_{\operatorname{ker} B(u)^{\perp}}}\right)^{-1} f_{i}(u)$ for $i=r+1, \ldots, n$.

Lemma G.2. Let $V$ and $W$ be finite dimensional, continuous $K$-modules and assume that for $x \in X, B(x) \in \operatorname{Hom}_{K}(V, W)$ is surjective and the map $x \mapsto B(x)$ is continuous. Then $x \mapsto \operatorname{ker} B(x)$ is a $K$-vector bundle over $X$.

Proof. Let $x_{o} \in X$; we must show that there is a neighborhood $U_{1}$ of $x_{o}$ and for all $u \in U_{1}$ a $K$-module isomorphism $T(u)$ of $\operatorname{ker} B\left(x_{o}\right)$ onto ker $B(u)$ such that the map $u \mapsto T(u)$ is continuous from $U_{1}$ to $H_{o m}\left(\operatorname{ker} B\left(x_{o}\right), V\right)$. To that end, let $M \subset V$ be a $K$-invariant subspace of $V$ such that $B\left(x_{o}\right)$ is a $K$-isomorphism of $M$ onto $W$. Then there exists $U \subset X$ an open neighborhood of $x_{o}$ such that $B(u)_{\mid M}$ is invertible for $u \in U$. Set $S(u)=\left(B(u)_{\mid M}\right)^{-1}$ on $B(u) M=W=B(u) V$ for $u \in U$. If $v \in \operatorname{ker} B\left(x_{0}\right)$ and if $u \in U$ then

$$
B(u) v=B(u) S(u) B(u) v
$$

so

$$
B(u)(I-S(u) B(u)) v=0 .
$$

Thus $I-S(u) B(u)$ maps $\operatorname{ker} B\left(x_{o}\right)$ to $\operatorname{ker} B(u)$ for $u \in U$. This map is the identity for $u=x_{o}$, so it is a $K$-isomorphism for $u \in U_{1} \subset U$ with $U_{1}$ open in $U$.

Proposition G.3. If $(\sigma, V)$ is a continuous family of admissible $(\mathfrak{g}, K)$-modules over a metric space $X$, if $(\mu, H)$ is a smoothable (see Definition [F.3) continuous family of admissible Hilbert representations of $G$ based on $X$ and if

$$
T:(\sigma, V) \rightarrow\left(d \mu, H_{K}\right)
$$

is a continuous family of injective $(\mathfrak{g}, K)$-module homomorphisms then there exists $(\lambda, W)$ a smoothable continuous family of Hilbert representations of $G$ based on $X$ such that $\left(d \lambda, W_{K}^{\infty}\right)$ and $(\sigma, V)$ are isomorphic as continuous families and a continuous family of injections of $(\lambda, W)$ into $(\mu, H)$.

Proof. If $x \in X$ then set $\langle\ldots, \ldots\rangle_{x}=T_{x}^{*}\langle\ldots, \ldots\rangle$ (where $\langle\ldots, \ldots\rangle$ is the inner product on $H$ ). If $\gamma \in \widehat{K}, x \in X$ let $e_{1}^{\gamma}(x), \ldots, e_{n_{\gamma}}^{\gamma}(x)$ be the orthonormal basis as in Lemma G.1 corresponding to the restriction of $\langle\ldots, \ldots\rangle_{x}$ to $V(\gamma)$. Then $\left\{e_{i}^{\gamma}(x)\right\}_{i, \gamma \in \hat{K}_{V}}$ with $\hat{K}_{V}=\{\gamma \in \hat{K} \mid V(\gamma) \neq 0\}$ is an orthonormal basis of $V$. Set

$$
f_{i}^{\gamma}(x)=T_{x}\left(e_{i}^{\gamma}(x)\right)
$$


then $\left\{f_{i}^{\gamma}(x)\right\}$ is an orthonormal basis of $T_{x} V$ for $x \in X$. If $v \in H$ then set

$$
P_{\gamma}(x) v=\sum_{i=1}^{n_{\gamma}}\left\langle v, f_{i}^{\gamma}(x)\right\rangle f_{i}^{\gamma}(x) .
$$

Note that the map

$$
x \mapsto P_{\gamma}(x)
$$

is strongly continuous from $X$ to $\operatorname{Hom}_{K}(H, H(\gamma)$ ) (the continuous $K$ homomorphisms). Define

$$
P(x) v=\sum P_{\gamma}(x) v .
$$

Then $P(x)$ is the orthogonal projection of $H$ onto the closure of $T_{x} V$ in $H$. Thus in particular $\|P(x)\|=1$. We assert that

$$
x \mapsto P(x)
$$

is strongly continuous from $X$ to the bounded operators on $H$. To this end, let $v \in H$ be a unit vector and $x_{o} \in X$. Let $\varepsilon>0$ be given and let $F \subset \widehat{K}$ be such that

$$
\left\|\sum_{\gamma \notin F} v(\gamma)\right\|<\frac{\varepsilon}{4},
$$

then since

$$
P(x) \sum_{\gamma \in F} v(\gamma)=\sum_{\gamma \in F} P_{\gamma}(x) v(\gamma),
$$

there exists an open neighborhood, $U$, of $x_{o}$ in $X$ such that

$$
\left\|\left(P(x)-P\left(x_{o}\right)\right) \sum_{\gamma \in F} v(\gamma)\right\|<\frac{\varepsilon}{2} .
$$

Thus

$$
\begin{gathered}
\left\|\left(P(x)-P\left(x_{o}\right)\right) v\right\| \leq\left\|\left(P(x)-P\left(x_{o}\right)\right) \sum_{\gamma \in F} v(\gamma)\right\|+\left\|\left(P(x)-P\left(x_{o}\right)\right) \sum_{\gamma \notin F} v(\gamma)\right\| \\
\leq\left\|\left(P(x)-P\left(x_{o}\right)\right) \sum_{\gamma \in F} v(\gamma)\right\|+2\left\|\sum_{\gamma \notin F} v(\gamma)\right\|<\varepsilon .
\end{gathered}
$$

Let $\nu_{x}(g)$ be the action of $G$ on $P(x) H$. Define $L(x, y): P(y) H \rightarrow P(x) H$

$$
L(x, y) f_{i}^{\gamma}(y)=f_{i}^{\gamma}(x) .
$$

Then $L(x, y)$ is a unitary operator and a $K$-module equivalence. Furthermore,

$$
x, y \mapsto L(x, y) P(y)
$$

is strongly continuous (use a slight modification of the argument for the strong continuity of $P(x))$. Fix $x_{o} \in X$ and set $W=P\left(x_{o}\right) H$ and

$$
\lambda_{x}(g)=L\left(x_{o}, x\right) \nu_{x}(g) L\left(x, x_{o}\right) .
$$

To complete the proof we need to show that $(\mu, W)$ is smoothable. Let $\omega$ be a compact subset of $X$ and $u \in U(\mathfrak{g})$; then there exist $C_{\omega}$ and $n_{\omega}$ such that if $v \in H^{\infty}$ then the definition of smoothable says

$$
\left\|d \mu_{x}(u) v\right\| \leq C_{\omega}\left\|d \mu_{x}\left(1+C_{K}\right)^{n_{\omega}} v\right\| .
$$


Now, if $v \in W^{\infty}$ then $v=P_{x_{o}} w$ with $w \in H^{\infty}$. So

$$
\begin{gathered}
\left\|d \lambda_{x}(u) v\right\|=\left\|L\left(x_{o}, x\right) d \nu_{x}(u) L\left(x, x_{o}\right) P_{x_{0}} w\right\| \\
=\left\|d \nu_{x}(u) L\left(x, x_{o}\right) P_{x_{0}} w\right\| \leq C_{\omega}\left\|d \nu_{x}\left(1+C_{K}\right)^{n_{\omega}} L\left(x, x_{o}\right) P_{x_{0}} w\right\| \\
=C_{\omega}\left\|L\left(x_{o}, x\right) d \nu_{x}\left(1+C_{K}\right)^{n_{\omega}} L\left(x, x_{o}\right) P_{x_{0}} w\right\| \\
=C_{\omega}\left\|d \lambda_{x}\left(1+C_{K}\right)^{n_{\omega}} P_{x_{0}} w\right\| .
\end{gathered}
$$

Using similar methods we have

Proposition G.4. If $(\sigma, V)$ is a continuous family of admissible $(\mathfrak{g}, K)$-modules over a compact contractible metric space $X$, if $(\mu, H)$ is a continuous, smoothable family of admissible Hilbert representations of $G$ based on $X$ and if

$$
T:\left(d \mu, H_{K}\right) \rightarrow(\sigma, V)
$$

is a continuous family of surjective $(\mathfrak{g}, K)$-module homomorphisms then there exists $(\lambda, W)$ a smoothable, continuous family of Hilbert representations of $G$ based on $X$ such that $\left(d \lambda, W_{K}^{\infty}\right)$ and $(\sigma, V)$ are isomorphic as continuous families and a continuous family of surjections of $(\mu, H)$ onto $(\lambda, W)$. Furthermore, if $(\mu, H)$ is smoothable then so is $(\lambda, W)$.

Proof. The proof follows the same lines as the previous theorem. Let for each $x \in X$,

$$
B_{\gamma}(x)=\left.T_{x}\right|_{H(\gamma)}
$$

Let $r_{\gamma}=\operatorname{dim} V(\gamma), m_{\gamma}=\operatorname{dim} H(\gamma)$. Then Lemma G.1 implies that for each $x \in X$ there exists an orthonormal basis of $H(\gamma),\left\{e_{i}^{\gamma}(x)\right\}$ with respect to the inner product, $\langle\ldots, \ldots\rangle$ on $H$ such that $\left\{e_{i}^{\gamma}(x)\right\}_{i>r_{\gamma}}$ is a basis of $\operatorname{ker} B_{\gamma}(x)$ and $x \mapsto e_{\gamma}^{i}(x)$ is continuous. Let $f_{i}^{\gamma}(x)=B_{\gamma}(x) e_{i}^{\gamma}(x)$ and for each $x \in X$ define an inner product, $\langle\ldots, \ldots\rangle_{x}$ on $V$ by declaring that $\left\{f_{i}^{\gamma}(x)\right\}$ is an orthonormal basis. Set

$$
P_{\gamma}(v)=\sum_{i=1}^{r_{\gamma}}\left\langle v, e_{i}^{\gamma}(x)\right\rangle e_{i}^{\gamma}(x)
$$

and $P(x)=\sum P_{\gamma}(x)$. Essentially the same argument as in the proof of the preceding theorem shows that map $x \mapsto P(x)$ is strongly continuous. Also, $T_{x}$ : $P(x) H_{K} \rightarrow V$ is unitary relative to $\langle\ldots, \ldots\rangle_{x}$ and an equivalence of representations of $K$. Let $H_{1, x}$ be the closure in $H$ of $\operatorname{ker} T_{x}$ for $x \in X$. Then, as a Hilbert space, under $T_{x} H / H_{1, x} \cong P(x) H$. Since $\operatorname{ker} T_{x}$ consists of analytic vectors $H_{1, x}$ is $G$-invariant. This defines a Hilbert representation, $\gamma_{x}$, on $P(x) H$. Which in turn defines a Hilbert representation, $\nu_{x}$, of $G$ on the Hilbert space completion of $V$, $Z_{x}$. Let $L(x, y): Z_{y} \rightarrow Z_{x}$ be defined by $L(x, y) f_{i}^{\gamma}(y)=f_{i}^{\gamma}(x)$. Then $L(x, y)$ defines a unitary $K$-isomorphism of $\left(\left.\nu_{y}\right|_{K}, Z_{y}\right)$ with $\left(\left.\nu_{x}\right|_{K}, Z_{x}\right)$. Fix $x_{o}$ in $X$ and let $W=Z_{x_{o}}$ and $\lambda_{x}(g)=L\left(x_{o}, x\right) \nu_{x}(g) L\left(x, x_{o}\right)$. As in the preceding theorem, we have defined a Hilbert family globalizing $(\sigma . V)$.

We now assume that $(\mu, H)$ is smoothable. If $v \in P_{x} H^{\infty}$ then

$$
d \mu_{x}(u) v=d \gamma_{x}(u) v+\left(I-P_{x}\right) d \mu_{x}(u) v .
$$

Thus, if $\omega$ is a compact subset of $X$ then

$$
\begin{gathered}
\left\|d \gamma_{x}(u) v\right\| \leq\left\|d \mu_{x}(u) v\right\| \\
\leq C_{u, \omega}\left\|d \mu_{x}\left(1+C_{K}\right)^{l} v\right\|=C_{u, \omega}\left\|d \gamma_{x}\left(1+C_{K}\right)^{l} v\right\| .
\end{gathered}
$$


Let $M(x, y): P(x) H \rightarrow P(y) H$ be given by

$$
M(x, y) e_{i}^{\gamma}(y)=e_{i}^{\gamma}(x) .
$$

Fix $x_{o} \in X$. Then the family can be defined as $\delta_{x}(g)=M\left(x_{o}, x\right) \gamma_{x}(g) M\left(x, x_{o}\right) v$ for $v \in P\left(x_{o}\right) H$. Setting $W=P\left(x_{o}\right) H$ then $(\delta, U)$ is an isomorphic continuous family to $(\lambda, W)$. We show that this family is smoothable; let $u \in U(\mathfrak{g})$. Then if $v \in U$ and $\omega$ is a compact subset of $X$ then

$$
\begin{gathered}
\left\|d \delta_{x}(u) v\right\|=\left\|M\left(x_{o}, x\right) d \gamma_{x}(u) M\left(x, x_{o}\right) v\right\|=\left\|d \gamma_{x}(u) M\left(x, x_{o}\right) v\right\| \\
\leq C_{v, \omega}\left\|d \gamma_{x}\left(1+C_{K}\right)^{l} M\left(x, x_{o}\right) v\right\|=C_{u, \omega}\left\|M\left(x_{o}, x\right) d \gamma_{x}\left(1+C_{K}\right)^{l} M\left(x, x_{o}\right) v\right\| \\
=C_{u, \omega} \| d \delta_{x}\left(\left(1+C_{K}\right)^{l} v \| .\right.
\end{gathered}
$$

\section{REFERENCES}

[A] M. F. Atiyah, K-theory, W. A. Benjamin, Inc., New York-Amsterdam, 1967. Lecture notes by D. W. Anderson. MR0224083

[BK] Joseph Bernstein and Bernhard Krötz, Smooth Fréchet globalizations of Harish-Chandra modules, Israel J. Math. 199 (2014), no. 1, 45-111, DOI 10.1007/s11856-013-0056-1. MR3219530

[BW] A. Borel and N. Wallach, Continuous cohomology, discrete subgroups, and representations of reductive groups, 2nd ed., Mathematical Surveys and Monographs, vol. 67, American Mathematical Society, Providence, RI, 2000, DOI 10.1090/surv/067. MR.1721403

[G1] Alexandre Grothendieck, Sur certains espaces de fonctions holomorphes. II (French), J. Reine Angew. Math. 192 (1953), 77-95, DOI 10.1515/crll.1953.192.77. MR62335

[G2] A. Grothendieck, Topological vector spaces, Notes on Mathematics and its Applications, Gordon and Breach Science Publishers, New York-London-Paris, 1973. Translated from the French by Orlando Chaljub. MR0372565

[H] Sigurdur Helgason, Some results on invariant differential operators on symmetric spaces, Amer. J. Math. 114 (1992), no. 4, 789-811, DOI 10.2307/2374798. MR.1175692

[HOW] Jing-Song Huang, Toshio Oshima, and Nolan Wallach, Dimensions of spaces of generalized spherical functions, Amer. J. Math. 118 (1996), no. 3, 637-652. MR1393264

[KR] B. Kostant and S. Rallis, Orbits and representations associated with symmetric spaces, Amer. J. Math. 93 (1971), 753-809, DOI 10.2307/2373470. MR311837

[RS] Michael Reed and Barry Simon, Methods of modern mathematical physics. I, 2nd ed., Academic Press, Inc. [Harcourt Brace Jovanovich, Publishers], New York, 1980. Functional analysis. MR751959

[T] François Trèves, Topological vector spaces, distributions and kernels, Academic Press, New York-London, 1967. MR0225131

[VdN] Vincent van der Noort, Analytic parameter dependence of Harish-Chandra modules for real reductive groups, a family affair, Thesis, University of Utrecht, 2009, http://dspace.library.uu.nl/handle/1874/37141.

[W1] Nolan R. Wallach, Harmonic analysis on homogeneous spaces, Pure and Applied Mathematics, No. 19, Marcel Dekker, Inc., New York, 1973. MR.0498996

[RRG] Nolan R. Wallach, Real reductive groups. II, Pure and Applied Mathematics, vol. 132, Academic Press, Inc., Boston, MA, 1992. MR1170566

[W] Garth Warner, Harmonic analysis on semi-simple Lie groups. I, Die Grundlehren der mathematischen Wissenschaften, Band 188, Springer-Verlag, New York-Heidelberg, 1972. MR0498999

Department of Mathematics, University of California San Diego, La Jolla, CaliforNIA

Email address: nwallach@ucsd.edu 